\title{
COMPUTING THE STACKELBERG/NASH EQUILIBRIA USING THE EXTRAPROXIMAL METHOD: CONVERGENCE ANALYSIS AND IMPLEMENTATION DETAILS FOR MARKOV CHAINS GAMES
}

\author{
Kristal K. TREJO ${ }^{a}$, Julio B. CLEMPNER ${ }^{b}$, AleXANder S. POZNYAK ${ }^{a *}$ \\ ${ }^{a}$ Department of Automatic Control, Center for Research and Advanced Studies \\ Av. IPN 2508, Col. San Pedro Zacatenco, 07360 Mexico City, Mexico \\ e-mail: \{apoznyak, ktrejo\}@etrl.cinvestav.mx \\ ${ }^{b}$ Center for Economics, Management and Social Research, National Polytechnic Institute \\ Lauro Aguirre 120, col. Agricultura, Del. Miguel Hidalgo, Mexico City, 11360, Mexico \\ e-mail: julio@clempner. name
}

\begin{abstract}
In this paper we present the extraproximal method for computing the Stackelberg/Nash equilibria in a class of ergodic controlled finite Markov chains games. We exemplify the original game formulation in terms of coupled nonlinear programming problems implementing the Lagrange principle. In addition, Tikhonov's regularization method is employed to ensure the convergence of the cost-functions to a Stackelberg/Nash equilibrium point. Then, we transform the problem into a system of equations in the proximal format. We present a two-step iterated procedure for solving the extraproximal method: (a) the first step (the extra-proximal step) consists of a "prediction" which calculates the preliminary position approximation to the equilibrium point, and (b) the second step is designed to find a "basic adjustment" of the previous prediction. The procedure is called the "extraproximal method" because of the use of an extrapolation. Each equation in this system is an optimization problem for which the necessary and efficient condition for a minimum is solved using a quadratic programming method. This solution approach provides a drastically quicker rate of convergence to the equilibrium point. We present the analysis of the convergence as well the rate of convergence of the method, which is one of the main results of this paper. Additionally, the extraproximal method is developed in terms of Markov chains for Stackelberg games. Our goal is to analyze completely a three-player Stackelberg game consisting of a leader and two followers. We provide all the details needed to implement the extraproximal method in an efficient and numerically stable way. For instance, a numerical technique is presented for computing the first step parameter $(\lambda)$ of the extraproximal method. The usefulness of the approach is successfully demonstrated by a numerical example related to a pricing oligopoly model for airlines companies.
\end{abstract}

Keywords: extraproximal method, Stackelberg games, convergence analysis, Markov chains, implementation.

\section{Introduction}

The extraproximal approach (Antipin, 2005) can be considered a natural extension of the proximal and gradient optimization methods used for solving the more difficult problems of finding an equilibrium point in game theory. The simplest and most natural approach to implement the proximal method is to use a simple iteration by omitting the prediction step. However, as shown by Antipin (2005), this approach fails. A more versatile procedure would be to perform an "ex-

*Corresponding author traproximal step" i.e., gathering certain information on the future development of the process. Using this information, it is possible to execute the "principle step" based on the preliminary position. It seems natural to call this procedure an "extraproximal method" (because of the use of extrapolation (Antipin, 2005)). Along with the extra-gradient technique (Poznyak, 2009), the extraproximal method can be considered a natural extension of the proximal and gradient optimization approaches to resolve more complicated game problems, such as the Stackelberg-Nash game.

Our goal is to analyze a three-player Stackelberg 
game (von Stackelberg, 1934), a leader and two followers, for a class of ergodic controllable finite Markov chains (Poznyak et al., 2000) using the extraproximal method (Antipin, 2005). The leader commits first to a strategy $x$ in the Markov chains game. Then, the followers realize a Nash solution (Clempner and Poznyak, 2011) obtaining $\varphi_{l}(\cdot, \cdot \mid x) \quad(l=1,2)$ as the cost functions. The optimal actions $x$ of the leader are then chosen to minimize its payoff $\varphi_{0}(\cdot, \cdot \mid x)$. The main concern about Stackelberg games is as follows: the highest leader payoff is obtained when the followers always reply in the best possible way for the leader (this payoff is at least as high as any Nash payoff). For ground-breaking works in the Stackelberg game, see those by Bos (1986; 1991), Harris and Wiens (1980), Merril and Schneider (1966) or von Stackelberg (1934). Surveys can be found in the works of Breitmoser (2012), De Fraja and Delbono (1990), Nett (1993) or Vickers and Yarrow. (1998).

In this paper we present the extraproximal method for computing the Stackelberg/Nash equilibria in a class of ergodic controlled finite Markov chains games. We exemplify the original game formulation in terms of coupled nonlinear programming problems implementing the Lagrange principle. In addition, Tikhonov's regularization method is employed to ensure the convergence of the cost-functions to a Stackelberg/Nash equilibrium point. The Tikhonov regularization is one of the most popular approaches to solve discrete ill-posed problems represented in the form of a non-obligatory strict convex function. Then, we transform the problem to a system of equations in the proximal format.

We present a two-step iterated procedure for solving the extraproximal method: (a) the first step (the extra-proximal step) consists of a "prediction" which calculates the preliminary position approximation to the equilibrium point, and (b) the second step is designed to find a "basic adjustment" of the previous prediction. Each equation in this system is an optimization problem for which the necessary condition for a minimum is solved using a quadratic programming method, instead of the iterating projectional gradient method given by Moya and Poznyak (2009). Iterating these steps, we obtain a new quick procedure which leads to a simple and logically justified computational realization: at each iteration of both sub-steps of the procedure, the functional of the game decreases and finally converges to a Stackelberg/Nash equilibrium point (Trejo et al., 2015).

We present the analysis of the convergence as well the rate of convergence of the method. Additionally, the extraproximal method is developed in terms of Markov chains for Stackelberg games consisting of a leader and two followers. We provide all the details needed to implement the extraproximal method in an efficient and numerically stable way. For instance, a numerical method is presented for computing the first step parameter $(\lambda)$ of the extraproximal method. The usefulness of the method is successfully demonstrated by a numerical example related to a pricing oligopoly model for airlines companies that choose to offer limited airline seats for specific routes.

The paper is organized as follows. The next section presents the preliminaries needed to understand the rest of the paper. The formulation of the problem for the $\mathcal{N}$-player game and the general format iterative version of the extraproximal method is presented in Section 2. A three-player Stackelberg game for a class of ergodic controllable finite Markov chains using the extraproximal method is given in Section 4. Section 5 includes the analysis of the converge of the extraproximal method and proves the convergence rates, which is one of the main results of this paper. A numerical example related to a pricing oligopoly model for airline companies validates the proposed extraproximal method in Section 6. Final comments are outlined in Section 7.

\section{Controllable Markov chains}

A controllable Markov chain (Clempner and Poznyak, 2014; Poznyak et al., 2000) is a quadruple $M C=$ $\{S, A, \Upsilon, \Pi\}$, where $S$ is a finite set of states, $S \subset \mathbb{N}$, endowed with discrete topology; $A$ is the set of actions, which is a metric space. For each $s \in S, A(s) \subset A$ is the non-empty set of admissible actions at state $s \in S$. Without loss of generality we may take $A=\cup_{s \in S} A(s)$; $\Upsilon=\{(s, a) \mid s \in S, a \in A(s)\}$ is the set of admissible state-action pairs, which is a measurable subset of $S \times A$; $\Pi(k)=\left[\pi_{(i, j \mid k)}\right]$ is a stationary transition controlled matrix, where

$$
\begin{aligned}
& \pi_{(i, j \mid k)} \\
& \stackrel{\equiv}{ } P\left(s(n+1)=s_{(j)} \mid s(n)=s_{(i)}, a(n)=a_{(k)}\right)
\end{aligned}
$$

representing the probability associated with the transition from state $s_{(i)}$ to state $s_{(j)}$ under an action $a_{(k)} \in A\left(s_{(i)}\right)$ $(k=1, \ldots, M)$ at time $n=0,1, \ldots$.

The dynamics of the game for Markov chains are described as follows. The game consists of $(\mathcal{N}+1)$ players (denoted by $l=\overrightarrow{0, \mathcal{N}}$ ) and begins at the initial state $s^{l}(0)$ which (as well as the states further realized by the process) is assumed to be completely measurable. Each player $l$ is allowed to randomize, with distribution $d_{(k \mid i)}^{l}(n)$, over the pure action choices $a_{(k)}^{l} \in A^{l}\left(s_{(i)}^{l}\right)$, $i=\overline{1, N_{l}}$ and $k=\overline{1, M_{l}}$. The leader corresponds to $l=0$ and followers to $l=\overline{1, \mathcal{N}}$. At each fixed strategy of the leader $d_{(k \mid i)}^{0}(n)$, the followers make the strategy selection trying to realize a Nash equilibrium. Below we will consider only stationary strategies $d_{(k \mid i)}^{l}(n)=d_{(k \mid i)}^{l}$. 
These choices induce the state distribution dynamics

$$
\begin{aligned}
& P^{l}\left(s^{l}(n+1)=s_{\left(j_{l}\right)}\right) \\
& =\sum_{i_{l}=1}^{N_{l}}\left(\sum_{k_{l}=1}^{M_{l}} \pi_{\left(i_{l}, j_{l} \mid k_{l}\right)}^{l} d_{\left(k_{l} \mid i_{l}\right)}^{l}\right) P^{l}\left(s^{l}(n)=s_{\left(i_{l}\right)}\right) .
\end{aligned}
$$

In the ergodic case (when all Markov chains are ergodic for any stationary strategy $\left.d_{(k \mid i)}^{l}\right)$ the distributions $P^{l}\left(s^{l}(n+1)=s_{\left(j_{l}\right)}\right)$ exponentially quickly converge to their limits $P^{l}\left(s=s_{(i)}\right)$ satisfying

$$
\begin{aligned}
& P^{l}\left(s^{l}=s_{\left(j_{l}\right)}\right) \\
& =\sum_{i_{l}=1}^{N_{l}}\left(\sum_{k_{l}=1}^{M_{l}} \pi_{\left(i_{l}, j_{l} \mid k_{l}\right)}^{l} d_{\left(k_{l} \mid i_{l}\right)}^{l}\right) P^{l}\left(s^{l}=s_{\left(i_{l}\right)}\right) .
\end{aligned}
$$

For any player $l$, his/her individual rationality is the player's cost function $J^{l}$ of any fixed policy $d^{l}$, defined over all possible combinations of states and actions, and indicates the expected value when taking action $a^{l}$ in state $s^{l}$ and following policy $d^{l}$ thereafter. The $J$-values can be expressed by

$$
\mathbf{J}^{l}\left(c^{l}\right):=\sum_{i_{l}, k_{l}} W_{\left(i_{l}, k_{l}\right)}^{l} d_{\left(k_{l} \mid i_{l}\right)}^{l} P^{l}\left(s^{l}=s_{\left(i_{l}\right)}\right),
$$

where

$$
W_{\left(i_{l}, k_{l}\right)}^{l}=\sum_{j_{l}} J_{\left(i_{l}, j_{l}, k_{l}\right)}^{l} \pi_{\left(i_{l}, j_{l} \mid k_{l}\right)}^{l}
$$

the function $J_{\left(i_{l}, j_{l}, k_{l}\right)}^{l}$ being a constant at state $s_{\left(i_{l}\right)}$ when the action $a_{\left(k_{l}\right)}^{l}$ is applied. Then, the cost function of each player, depending on the states and actions of all participants, is given by the values $W_{\left(i_{1}, k_{1} ; \ldots ; i_{\mathcal{N}}, k_{\mathcal{N}}\right)}^{l}$, so that the "average cost function" $\mathbf{J}^{l}$ for each player $l$ in the stationary regime can be expressed as

$$
\begin{aligned}
& \mathbf{J}^{l}\left(c^{0}, \ldots, c^{\mathcal{N}}\right) \\
& \quad:=\sum_{i_{0}, k_{0}} \cdots \sum_{i_{\mathcal{N}, k_{\mathcal{N}}}} W_{\left(i_{1}, k_{1}, \ldots, i_{\mathcal{N}}, k_{\mathcal{N}}\right)}^{l} \prod_{l=0}^{\mathcal{N}} c_{\left(i_{l}, k_{l}\right)}^{l},
\end{aligned}
$$

where

$$
c^{l}:=\left\|c_{\left(i_{l}, k_{l}\right)}^{l}\right\|_{i_{l}=\overline{1, N_{l}} ; k_{l}=\overline{1, M_{l}}}
$$

is a matrix with elements

$$
c_{\left(i_{l}, k_{l}\right)}^{l}=d_{\left(k_{l} \mid i_{l}\right)}^{l} P^{l}\left(s^{l}=s_{\left(i_{l}\right)}\right)
$$

satisfying

$$
c^{l} \in C_{\mathrm{adm}}^{l}=\left\{\begin{array}{c}
c^{l}: \sum_{i_{l}, k_{l}} c_{\left(i_{l}, k_{l}\right)}^{l}=1, c_{\left(i_{l}, k_{l}\right)}^{l} \geq 0, \\
\sum_{k_{l}} c_{\left(j_{l}, k_{l}\right)}^{l}=\sum_{i_{l}, k_{l}} \pi_{\left(i_{l}, j_{l} \mid k_{l}\right)}^{l} c_{\left(i_{l}, k_{l}\right)}^{l} .
\end{array}\right.
$$

Notice that by (4) it follows that

$$
\begin{gathered}
P^{l}\left(s^{l}=s_{\left(i_{l}\right)}\right)=\sum_{k_{l}} c_{\left(i_{l}, k_{l}\right)}^{l}, \\
d_{\left(k_{l} \mid i_{l}\right)}^{l}=\frac{c_{\left(i_{l}, k_{l}\right)}^{l}}{\sum_{k_{l}} c_{\left(i_{l}, k_{l}\right)}^{l}} .
\end{gathered}
$$

In the ergodic case,

$$
\sum_{k_{l}} c_{\left(i_{l}, k_{l}\right)}^{l}>0
$$

for all $l=\overline{0, \mathcal{N}}$. The individual aim of each participant is

$$
\mathbf{J}^{l}\left(c^{l}\right) \rightarrow \min _{c^{l} \in C_{\text {adm }}^{l}} .
$$

Obviously, here we have a conflict situation which can be resolved by the Stackelberg-Nash equilibrium concept discussed in detail below.

\section{Formulation of the problem}

3.1. Stackelberg-Nash equilibrium concept. To simplify the descriptions, below let us introduce the new variables:

$$
x:=\operatorname{col} c^{(0)}, \quad X:=C_{\mathrm{adm}}^{(0)},
$$

$$
v^{l}:=\operatorname{col} c^{(l)}, \quad V^{l}:=C_{\mathrm{adm}}^{(l)} \quad(l=\overline{1, \mathcal{N}}) .
$$

Consider a non-zero sum game with a leader whose strategies are denoted by $x \in X$ and $\mathcal{N}$ followers with strategies $v^{l} \in V^{l}(l=\overline{1, \mathcal{N}})$. Denote by

$$
v=\left(v^{1}, \ldots, v^{\mathcal{N}}\right) \in V:=\bigotimes_{l=1}^{\mathcal{N}} V^{l}
$$

the joint strategy of the followers. The leader is assumed to anticipate the reactions of the followers. They are trying to reach one of the Nash equilibria for any fixed strategy $x$ of the leader, that is, to find a joint strategy $w^{*}=\left(w^{1 *}, \ldots, w^{\mathcal{N} *}\right) \in W$ satisfying for any admissible $x \in X, w^{l} \in W^{l}$ and any $l=\overline{1, \mathcal{N}}$ the system of inequalities (the Nash condition)

$$
\begin{gathered}
g_{l}\left(v^{l}, w^{\hat{l} *} \mid x\right) \leq 0 \quad \text { for any } v^{l} \in V^{l} \text { and all } l=\overline{1, \mathcal{N}}, \\
g_{l}\left(v^{l}, w^{\hat{l}} \mid x\right):=\varphi_{l}\left(w^{l}, w^{\hat{l}} \mid x\right)-\varphi_{l}\left(v^{l}, w^{\hat{l}} \mid x\right),
\end{gathered}
$$

where $w^{\hat{l}}$ is a strategy of the rest of the followers adjoint to $v^{l}$, namely,

$$
\begin{aligned}
w^{\hat{l}} & :=\left(v^{1}, \ldots, v^{l-1}, v^{l+1}, \ldots, v^{\mathcal{N}}\right) \\
& \in W^{\hat{\imath}}:=\bigotimes_{m=1, m \neq l}^{\mathcal{N}} V^{m}
\end{aligned}
$$


and

$$
w^{l}:=\arg \min _{v^{l} \in V^{l}} \varphi_{l}\left(v^{l}, w^{\hat{l}} \mid x\right)
$$

Here $\varphi_{l}\left(v^{l}, w^{\hat{l}} \mid x\right)$ is the cost-function of the player $l$, which plays the strategy $v^{l} \in V^{l}$, and the rest of the players-the strategy $w^{\hat{l}} \in W^{\hat{l}}$.

Lemma 1. The Nash equilibrium $\bar{w} \in W$ for the followers given a fixed strategy of the leader $x \in X$ (8) can be equivalently expressed in the joint format (Tanaka and Yokoyama, 1991),

$$
\begin{gathered}
\max _{v \in W} g(v, \bar{w} \mid x) \leq 0, \\
g(v, w \mid x):=\sum_{l=1}^{\mathcal{N}}\left[\varphi_{l}\left(w^{l}, w^{\hat{l}} \mid x\right)-\varphi_{l}\left(v^{l}, w^{\hat{l}} \mid x\right)\right], \\
\varphi_{l}\left(w^{l}, w^{\hat{l}} \mid x\right):=\min _{z^{l} \in W^{l}} \varphi_{l}\left(z^{l}, w^{\hat{l}} \mid x\right), \\
v^{l} \in W^{l}, \quad w \in W:={\underset{\bigotimes}{l=1}}_{l=1}^{\mathcal{N}} W^{l} .
\end{gathered}
$$

Proof. Summing (8) implies (9). Conversely, taking $v^{m}=w^{m}$ for all $m \neq l$ in (9), which is valid for any admissible $v^{l}$, we obtain (8).

Notice that the condition $g(v, \bar{w} \mid x) \leq 0$ (9) is equivalent to

$$
\max _{v \in V}\{g(v, \bar{w} \mid x)\} \leq 0
$$

for any fixed $v \in V$ and any $x \in X$.

Let $f(x, w)(x \in X, w \in W)$ be the cost function of the leader. The functions of the followers $\varphi_{l}\left(v^{l}, w^{\hat{l}} \mid x\right)$ $(l=\overline{1, \mathcal{N}})$ and the function of the leader $f(x, w)$ are assumed to be convex in all their arguments.

Definition 1. A strategy $x^{*} \in X$ of the leader together with the collection $w^{*} \in W$ of the followers is said to be a Stackelberg-Nash equilibrium if

$$
\left(x^{*}, w^{*}\right) \in \operatorname{Arg} \min _{x \in X} \max _{v \in V, w \in W}\{f(x, w) \mid g(v, w \mid x) \leq 0\} .
$$

3.2. Regularized Lagrange principle application. Applying the Lagrange principle (see, for example, Poznyak et al., 2000) for Definition 1, we may conclude that (10) can be rewritten as

$$
\begin{gathered}
\left(x^{*}, w^{*}\right) \in \operatorname{Arg} \min _{x \in X} \max _{v \in V, w \in W, \lambda \geq 0} \mathcal{L}(x, v, w, \lambda), \\
\mathcal{L}(x, v, w, \lambda):=f(x, w)+\lambda g(v, w \mid x) .
\end{gathered}
$$

The approximative solution obtained by the Tikhonov regularization (see Poznyak et al., 2000) is given by

$$
\begin{aligned}
& \left(x_{\delta}^{*}, w_{\delta}^{*}\right)=\arg \min _{x \in X} \max _{v \in V, w \in W, \lambda \geq 0} \mathcal{L}_{\delta}(x, v, w, \lambda), \\
& \mathcal{L}_{\delta}(x, v, w, \lambda):=f_{\delta}(x, w)+\lambda g_{\delta}(v, w \mid x)-\frac{\delta}{2} \lambda^{2},
\end{aligned}
$$

where $\delta>0$ and

$$
\begin{aligned}
f_{\delta}(x, w) & :=f(x, w)+\frac{\delta}{2}\|x\|^{2}, \\
g_{\delta}(v, w \mid x) & =g(v, w \mid x)-\frac{\delta}{2}\left(\|v\|^{2}+\|w\|^{2}\right)
\end{aligned}
$$

Notice that with $\delta>0$ the functions considered become strictly convex providing the uniqueness of the discussed conditional optimization problem (12). For $\delta=$ 0 we have (11). Notice also that the Lagrange function in (12) satisfies the saddle-point condition (Poznyak, 2009), namely, for all $x \in X$ and $\lambda \geq 0$ we have

$$
\begin{aligned}
\mathcal{L}_{\delta}\left(x_{\delta}^{*}, v_{\delta}, w_{\delta}, \lambda_{\delta}\right) & \leq \mathcal{L}_{\delta}\left(x_{\delta}^{*}, v_{\delta}^{*}, w_{\delta}^{*}, \lambda_{\delta}^{*}\right) \\
& \leq \mathcal{L}_{\delta}\left(x_{\delta}, v_{\delta}^{*}, w_{\delta}^{*}, \lambda_{\delta}^{*}\right)
\end{aligned}
$$

3.3. Proximal format. In the proximal format (see Antipin, 2005) the relation (12) can be expressed as

$$
\begin{aligned}
& \lambda_{\delta}^{*}=\arg \max _{\lambda \geq 0}\left\{-\frac{1}{2}\left\|\lambda-\lambda_{\delta}^{*}\right\|^{2}+\gamma \mathcal{L}_{\delta}\left(x_{\delta}^{*}, v_{\delta}^{*}, w_{\delta}^{*}, \lambda\right)\right\}, \\
& x_{\delta}^{*}=\arg \min _{x \in X}\left\{\frac{1}{2}\left\|x-x_{\delta}^{*}\right\|^{2}+\gamma \mathcal{L}_{\delta}\left(x, v_{\delta}^{*}, w_{\delta}^{*}, \lambda_{\delta}^{*}\right)\right\}, \\
& v_{\delta}^{*}=\arg \max _{v \in V}\left\{-\frac{1}{2}\left\|v-v_{\delta}^{*}\right\|^{2}+\gamma \mathcal{L}_{\delta}\left(x_{\delta}^{*}, v, w_{\delta}^{*}, \lambda_{\delta}^{*}\right)\right\}, \\
& w_{\delta}^{*}=\arg \max _{w \in W}\left\{-\frac{1}{2}\left\|w-w_{\delta}^{*}\right\|^{2}+\gamma \mathcal{L}_{\delta}\left(x_{\delta}^{*}, v_{\delta}^{*}, w, \lambda_{\delta}^{*}\right)\right\},
\end{aligned}
$$

where the solutions $x_{\delta}^{*}, v_{\delta}^{*}, w_{\delta}^{*}$ and $\lambda_{\delta}^{*}$ depend on the small parameters $\delta, \gamma>0$.

Define the extended variables

$$
\begin{gathered}
\tilde{x}:=(x) \in \tilde{X}:=X, \\
\tilde{y}:=\left(\begin{array}{c}
v \\
w \\
\lambda
\end{array}\right) \in \tilde{Y}:=V \times W \times \mathbb{R}^{+}, \\
\tilde{w}=\left(\begin{array}{c}
\tilde{w}_{1} \\
\tilde{w}_{2}
\end{array}\right) \in \tilde{X} \times \tilde{Y}, \\
\tilde{v}=\left(\begin{array}{c}
\tilde{v}_{1} \\
\tilde{v}_{2}
\end{array}\right) \in \tilde{X} \times \tilde{Y},
\end{gathered}
$$


and the functions

$$
\begin{aligned}
\tilde{\mathcal{L}}_{\delta}(\tilde{x}, \tilde{y}) & :=\mathcal{L}_{\delta}(x, v, w, \lambda), \\
\Psi_{\delta}(\tilde{w}, \tilde{v}) & :=\tilde{\mathcal{L}}_{\delta}\left(\tilde{w}_{1}, \tilde{v}_{2}\right)-\tilde{\mathcal{L}}_{\delta}\left(\tilde{v}_{1}, \tilde{w}_{2}\right) .
\end{aligned}
$$

For $\tilde{w}_{1}=\tilde{x}, \tilde{w}_{2}=\tilde{y}, \tilde{v}_{1}=\tilde{v}_{1}^{*}=\tilde{x}_{\delta}^{*}$ and $\tilde{v}_{2}=\tilde{v}_{2}^{*}=\tilde{y}_{\delta}^{*}$, we have

$$
\Psi_{\delta}(\tilde{w}, \tilde{v}):=\tilde{\mathcal{L}}_{\delta}\left(\tilde{x}, \tilde{y}_{\delta}^{*}\right)-\tilde{\mathcal{L}}_{\delta}\left(\tilde{x}_{\delta}^{*}, \tilde{y}\right) .
$$

In these variables the relation (15) can be represented in a "short format" as

$$
\tilde{v}^{*}=\arg \min _{\tilde{w} \in \tilde{X} \times \widetilde{Y}}\left\{\frac{1}{2}\left\|\tilde{w}-\tilde{v}^{*}\right\|^{2}+\gamma \Psi_{\delta}\left(\tilde{w}, \tilde{v}^{*}\right)\right\} .
$$

3.4. Extraproximal method. The extraproximal method for the conditional optimization problems (12) was suggested by Antipin (2005). We design the method for the static Stackelberg-Nash game in a general format.

The general format iterative version $(n=0,1, \ldots)$ of the extraproximal method with some fixed admissible initial values $\left(x_{0} \in X, v_{0} \in V, w_{0} \in W\right.$, and $\left.\lambda_{0} \geq 0\right)$ is as follows:

1. The first half-step (prediction):

$$
\begin{aligned}
& \bar{\lambda}_{n}=\arg \min _{\lambda \geq 0}\left\{\frac{1}{2}\left\|\lambda-\lambda_{n}\right\|^{2}-\gamma \mathcal{L}_{\delta}\left(x_{n}, v_{n}, w_{n}, \lambda\right)\right\}, \\
& \bar{x}_{n}=\arg \min _{x \in X}\left\{\frac{1}{2}\left\|x-x_{n}\right\|^{2}+\gamma \mathcal{L}_{\delta}\left(x, v_{n}, w_{n}, \bar{\lambda}_{n}\right)\right\}, \\
& \bar{v}_{n}=\arg \min _{v \in V}\left\{\frac{1}{2}\left\|v-v_{n}\right\|^{2}-\gamma \mathcal{L}_{\delta}\left(x_{n}, v, w_{n}, \bar{\lambda}_{n}\right)\right\}, \\
& \bar{w}_{n}=\arg \min _{w \in W}\left\{\frac{1}{2}\left\|w-w_{n}\right\|^{2}-\gamma \mathcal{L}_{\delta}\left(x_{n}, v_{n}, w, \bar{\lambda}_{n}\right)\right\},
\end{aligned}
$$

or, in the extended variables,

$$
\hat{v}_{n}=\arg \min _{\tilde{w} \in \widetilde{X} \times \widetilde{Y}}\left\{\frac{1}{2}\left\|\tilde{w}-\tilde{v}_{n}\right\|^{2}+\gamma \Psi_{\delta}\left(\tilde{w}, \tilde{v}_{n}\right)\right\} .
$$

2. The second (basic) half-step,

$$
\begin{aligned}
& \lambda_{n+1}=\arg \min _{\lambda \geq 0}\left\{\frac{1}{2}\left\|\lambda-\lambda_{n}\right\|^{2}-\gamma \mathcal{L}_{\delta}\left(\bar{x}_{n}, \bar{v}_{n}, \bar{w}_{n}, \lambda\right)\right\}, \\
& x_{n+1}=\arg \min _{x \in X}\left\{\frac{1}{2}\left\|x-x_{n}\right\|^{2}+\gamma \mathcal{L}_{\delta}\left(x, \bar{v}_{n}, \bar{w}_{n}, \bar{\lambda}_{n}\right)\right\}, \\
& v_{n+1}=\arg \min _{v \in V}\left\{\frac{1}{2}\left\|v-v_{n}\right\|^{2}-\gamma \mathcal{L}_{\delta}\left(\bar{x}_{n}, v, \bar{w}_{n}, \bar{\lambda}_{n}\right)\right\}, \\
& w_{n+1}=\arg \min _{w \in W}\left\{\frac{1}{2}\left\|w-w_{n}\right\|^{2}-\gamma \mathcal{L}_{\delta}\left(\bar{x}_{n}, \bar{v}_{n}, w, \bar{\lambda}_{n}\right)\right\}
\end{aligned}
$$

or, in the "short format",

$$
\tilde{v}_{n+1}=\arg \min _{\tilde{w} \in \tilde{X} \times \widetilde{Y}}\left\{\frac{1}{2}\left\|\tilde{w}-\tilde{v}_{n}\right\|^{2}+\gamma \Psi_{\delta}\left(\tilde{w}, \hat{v}_{n}\right)\right\} .
$$

Remark 1. The direct one-step procedure, when $\lambda_{n+1}=$ $\bar{\lambda}_{n}$, does not work (see the counter-example of Antipin (2005)).

\section{Markov format for the extraproximal method}

We consider a three-player Stackelberg game $(l=\overline{1, \mathcal{N}})$. Player $l$ should be understood to refer to players in a general expression of the game. In the remainder of this paper, the leader is designated as player (0) and the followers as players (1) and (2). Numbers are used to refer to players in a specific expression. We also assume that the number of strategies and actions that this description can take is finite and fixed. Here we will apply the iterative quadratic method providing a significantly quicker rate of convergence.

4.1. Cost functions and notation. The individual cost-functions of the followers and the leader are defined as follows.

\section{Cost-function for the leader:}

$$
\begin{aligned}
& \mathbf{J}^{(0)}\left(c^{(1)}, c^{(2)}, c^{(0)}\right) \\
& =\sum_{i_{1}, k_{1}} \sum_{i_{2}, k_{2}} \sum_{i_{0}, k_{0}} W_{\left(i_{1}, k_{1} ; i_{2}, k_{2} ; i_{0}, k_{0}\right)}^{(0)} c_{\left(i_{1}, k_{1}\right)}^{(1)} c_{\left(i_{2}, k_{2}\right)}^{(2)} c_{\left(i_{0}, k_{0}\right)}^{(0)} .
\end{aligned}
$$

\section{Cost-functions for the followers:}

Follower 1:

$$
\begin{aligned}
& \mathbf{J}^{(1)}\left(c^{(1)}, c^{(2)}, c^{(0)}\right) \\
& =\sum_{i_{1}, k_{1}} \sum_{i_{2}, k_{2}} \sum_{i_{0}, k_{0}} W_{\left(i_{1}, k_{1} ; i_{2}, k_{2} ; i_{0}, k_{0}\right)}^{(1)} c_{\left(i_{1}, k_{1}\right)}^{(1)} c_{\left(i_{2}, k_{2}\right)}^{(2)} c_{\left(i_{0}, k_{0}\right)}^{(0)} .
\end{aligned}
$$

Follower 2:

$$
\begin{aligned}
& \mathbf{J}^{(2)}\left(c^{(1)}, c^{(2)}, c^{(0)}\right) \\
& =\sum_{i_{1}, k_{1}} \sum_{i_{2}, k_{2}} \sum_{i_{0}, k_{0}} W_{\left(i_{1}, k_{1} ; i_{2}, k_{2} ; i_{0}, k_{0}\right)}^{(2)} c_{\left(i_{1}, k_{1}\right)}^{(1)} c_{\left(i_{2}, k_{2}\right)}^{(2)} c_{\left(i_{0}, k_{0}\right)}^{(0)} .
\end{aligned}
$$

For $n=0,1, \ldots$, let us define the vectors

$$
\begin{aligned}
& x_{n}=\operatorname{col} c^{(0)}(n), \\
& v_{n}=\left(\begin{array}{l}
\operatorname{col} c^{(1)}(n) \\
\operatorname{col} c^{(2)}(n)
\end{array}\right),
\end{aligned}
$$




$$
\begin{aligned}
w_{n} & =w^{\widehat{l}}=\left(\begin{array}{c}
\operatorname{col} c^{(\hat{1})}(n) \\
\operatorname{col} c^{(\hat{2})}(n)
\end{array}\right) \\
w^{l} & =\left(\begin{array}{c}
\operatorname{col} \stackrel{\circ}{(1)}^{(1)}(n) \\
\operatorname{col} c^{(2)}(n)
\end{array}\right) \\
& =\left(\begin{array}{c}
\operatorname{col} \arg \min _{z \in C_{a d m}^{(1)}} \mathbf{J}^{(1)}\left(z, c^{(\hat{2})}(n), x_{n}\right) \\
\operatorname{col} \arg \min _{z \in C_{a d m}^{(2)}} \mathbf{J}^{(2)}\left(c^{(\hat{1})}(n), z, x_{n}\right)
\end{array}\right) .
\end{aligned}
$$

Let us introduce for simplicity the following notation:

$$
\begin{aligned}
W_{\left(i_{1}, k_{1} ; i_{2}, k_{2} ; i_{0}, k_{0}\right)}^{(l)} & =W^{(l)} \\
c_{\left(i_{l}, k_{l}\right)}^{(l)} & =c^{(l)} \\
c^{(\hat{l})}\left(i_{l}, k_{l}\right) & =c^{(\hat{l})} \\
{\stackrel{\circ}{\left(i_{l}, k_{l}\right)}}^{(l)} & =c^{(l)} \\
\bar{c}_{\left(i_{l}, k_{l}\right)}^{(l)} & =\bar{c}^{(l)}
\end{aligned}
$$

For the leader we have

$$
f(x, w)=\sum_{i_{1}, k_{1}} \sum_{i_{2}, k_{2}} \sum_{i_{0}, k_{0}} W^{(0)} \stackrel{\circ}{c}^{(1)} \stackrel{c}{(2)}^{(2)} c^{(0)} .
$$

From

$$
\begin{aligned}
& \mathbf{J}^{(1)}\left(c^{(1)}, c^{(\hat{2})} \mid c^{(0)}\right) \leq \mathbf{J}^{(1)}\left(c^{(1)}, c^{(\hat{2})} \mid c^{(0)}\right), \\
& \mathbf{J}^{(2)}\left(c^{(2)}, c^{(\hat{1})} \mid c^{(0)}\right) \leq \mathbf{J}^{(2)}\left(c^{(2)}, c^{(\hat{1})} \mid c^{(0)}\right),
\end{aligned}
$$

we have

$$
\begin{aligned}
& \varphi_{l}\left(w^{l}, w^{\hat{l}} \mid x\right) \\
& =\mathbf{J}^{(1)}\left(c^{(1)}, c^{(\hat{2})} \mid c^{(0)}\right)+\mathbf{J}^{(2)}\left(c^{(2)}, c^{(\hat{1})} \mid c^{(0)}\right) \\
& =\sum_{i_{1}, k_{1}} \sum_{i_{2}, k_{2}} \sum_{i_{0}, k_{0}}\left[W^{(1)} c^{(1)} c^{(\hat{2})} c^{(0)}+W^{(2)} c^{(\hat{1})} c^{(2)} c^{(0)}\right],
\end{aligned}
$$

as well as

$$
\begin{aligned}
& \varphi_{l}\left(v^{l}, w^{\hat{l}} \mid x\right) \\
& =\mathbf{J}^{(1)}\left(c^{(1)}, c^{(\hat{2})} \mid c^{(0)}\right)+\mathbf{J}^{(2)}\left(c^{(2)}, c^{(\hat{1})} \mid c^{(0)}\right) \\
& =\sum_{i_{1}, k_{1}} \sum_{i_{2}, k_{2}} \sum_{i_{0}, k_{0}}\left[W^{(1)} c^{(1)} c^{(\hat{2})} c^{(0)}+W^{(2)} c^{(\hat{1})} c^{(2)} c^{(0)}\right] .
\end{aligned}
$$

For computing the Nash equilibrium point, we define the function $g(v, w, x)$ as follows:

$$
\begin{aligned}
& g(v, w \mid x) \\
& =\sum_{i_{1}, k_{1}} \sum_{i_{2}, k_{2}} \sum_{i_{0}, k_{0}}\left[W^{(1)} c^{(1)} c^{(\hat{2})} c^{(0)}+W^{(2)} c^{(\hat{1})} c^{(2)} c^{(0)}\right. \\
& \left.-W^{(1)} c^{(1)} c^{(\hat{2})} c^{(0)}-W^{(2)} c^{(\hat{1})} c^{(2)} c^{(0)}\right] .
\end{aligned}
$$

4.2. Tikhonov's regularization method. The regularized problem for (24) is defined as follows:

$$
f_{\delta}(x, w)=\sum_{i_{1}, k_{1}} \sum_{i_{2}, k_{2}} \sum_{i_{0}, k_{0}} W^{(0)}{ }^{(1)} c^{(2)} c^{(0)}+\frac{\delta}{2}\left\|c^{(0)}\right\|^{2},
$$

and for (27) we have

$$
\begin{aligned}
& g_{\delta}(v, w \mid x) \\
& =\sum_{i_{1}, k_{1}} \sum_{i_{2}, k_{2}} \sum_{i_{0}, k_{0}}\left[W^{(1)} c^{(1)} c^{(\hat{2})} c^{(0)}+W^{(2)} c^{(\hat{1})} c^{(2)} c^{(0)}\right. \\
& \left.\quad-W^{(1)} c^{(1)} c^{(\hat{2})} c^{(0)}-W^{(2)} c^{(\hat{1})} c^{(2)} c^{(0)}\right] \\
& \quad-\frac{\delta}{2}\left(\left\|\begin{array}{c}
c^{(1)} \\
c^{(2)}
\end{array}\right\|^{2}+\left\|\begin{array}{c}
c^{(\hat{1})} \\
c^{(\hat{2})}
\end{array}\right\|^{2}\right)
\end{aligned}
$$

4.3. Lagrange principle. Let us apply the Lagrange principle (Poznyak, 2009) with the Lagrange function

$$
\begin{aligned}
& \mathfrak{L}_{\delta}(x, v, w, \lambda) \\
& =f_{\delta}(x, w)+\lambda g_{\delta}(v, w, x)-\frac{\delta}{2} \lambda^{2} \\
& =\sum_{i_{1}, k_{1}} \sum_{i_{2}, k_{2}} \sum_{i_{0}, k_{0}}\left[W^{(0)} c^{(1)} c^{(2)} c^{(0)}\right. \\
& \quad+\lambda\left(W^{(1)} c^{(1)} c^{(\hat{2})} c^{(0)}+W^{(2)} c^{(\hat{1})} c^{(2)} c^{(0)}\right. \\
& \left.\left.\quad-W^{(1)} c^{(1)} c^{(2)} c^{(0)}-W^{(2)} c^{(\hat{1})} c^{(2)} c^{(0)}\right)\right] \\
& \quad-\lambda \frac{\delta}{2}\left(\left\|c^{(1)} c^{(2)}\right\|^{2}+\| \begin{array}{c}
c^{(\hat{1})} \|^{(\hat{2})} \\
2
\end{array}\right)+\frac{\delta}{2}\left\|c^{(0)}\right\|^{2}-\frac{\delta}{2} \lambda^{2} .
\end{aligned}
$$

4.4. Extrapoximal method for Markov chains. The procedure for solving the Stackelberg/Nash equilibrium point for the extraproximal method consists of the following "iterative rules" implementation.

\section{First half-step:}

1.a. For

$$
\bar{\lambda}_{n}=\arg \min _{\lambda \geq 0}\left\{\frac{1}{2}\left(\lambda-\lambda_{n}\right)^{2}-\gamma \mathfrak{L}_{\delta}\left(x_{n}, v_{n}, w_{n}, \lambda\right)\right\},
$$


we have

$$
\begin{aligned}
\bar{\lambda}_{n}= & {\left[\lambda_{n}+\gamma \sum_{i_{1}, k_{1}} \sum_{i_{2}, k_{2}} \sum_{i_{0}, k_{0}}\left(W^{(1)} c^{(1)} c^{(\hat{2})}(n) c^{(0)}(n)\right.\right.} \\
& +W^{(2)} c^{(\hat{1})}(n) c^{(2)} c^{(0)}(n) \\
& -W^{(1)} c^{(1)}(n) c^{(\hat{2})}(n) c^{(0)}(n) \\
& \left.-W^{(2)} c^{(\hat{1})}(n) c^{(2)}(n) c^{(0)}(n)\right) \\
& \left.-\gamma \frac{\delta}{2}\left(\left\|\begin{array}{l}
c^{(1)}(n) \\
c^{(2)}(n)
\end{array}\right\|^{2}\left\|\begin{array}{l}
(\hat{1}) \\
c^{(2)}(n)
\end{array}\right\|^{2}\right)\right]^{+} \\
& \times \frac{1}{1+\gamma \delta} .
\end{aligned}
$$

1.b. For

$$
\bar{x}_{n}=\arg \min _{x \in X}\left\{\frac{1}{2}\left\|x-x_{n}\right\|^{2}+\gamma \mathfrak{L}_{\delta}\left(x, v_{n}, w_{n}, \bar{\lambda}_{n}\right)\right\},
$$

we have

$$
\begin{aligned}
\bar{x}_{n}= & \arg \min _{x \in X}\left\{\left(\frac{1}{2}+\gamma \frac{\delta}{2}\right)\left\|c^{(0)}\right\|^{2}\right. \\
& +\gamma \sum_{i_{1}, k_{1}} \sum_{i_{2}, k_{2}} \sum_{i_{0}, k_{0}}\left[W^{(0)} c^{(1)} c^{(2)} c^{(0)}\right. \\
& +\bar{\lambda}_{n}\left(W^{(1)} c^{(1)} c^{(\hat{2})}(n) c^{(0)}\right. \\
& +W^{(2)} c^{(\hat{1})}(n) c^{(2)} c^{(0)}-W^{(1)} c^{(1)}(n) c^{(\hat{2})}(n) c^{(0)} \\
& \left.\left.-W^{(2)} c^{(\hat{1})}(n) c^{(2)}(n) c^{(0)}\right)\right] \\
& -c^{(0) T}(n) c^{(0)}+\frac{1}{2}\left\|c^{(0)}(n)\right\|^{2} \\
& -\gamma \bar{\lambda}_{n} \frac{\delta}{2}\left(\left\|c^{(1)}(n) c^{(2)}(n)+\right\| c^{(\hat{1})}(n) c^{(\hat{2})}(n) \|^{2}\right) \\
& \left.-\gamma \frac{\delta}{2} \bar{\lambda}_{n}^{2}\right\} .
\end{aligned}
$$

1.c. For

$$
\bar{v}_{n}=\arg \min _{v \in V}\left\{\frac{1}{2}\left\|v-v_{n}\right\|^{2}-\gamma \mathfrak{L}_{\delta}\left(x_{n}, v, w_{n}, \bar{\lambda}_{n}\right)\right\},
$$

we have

$$
\bar{v}_{n}=\arg \min _{v \in V}\left\{\left(\frac{1}{2}+\gamma \bar{\lambda}_{n} \frac{\delta}{2}\right)\left\|\begin{array}{c}
c^{(1)} \\
c^{(2)}
\end{array}\right\|^{2}\right.
$$

$$
\begin{aligned}
& -\left(\begin{array}{l}
c^{(1)} \\
c^{(2)}
\end{array}\right)^{T}\left(\begin{array}{l}
c^{(1)}(n) \\
c^{(2)}(n)
\end{array}\right) \\
& +\gamma \bar{\lambda}_{n} \sum_{i_{1}, k_{1}} \sum_{i_{2}, k_{2}} \sum_{i_{0}, k_{0}}\left[W^{(1)} c^{(1)} c^{(\hat{2})}(n) c^{(0)}(n)\right. \\
& +W^{(2)} c^{(\hat{1})}(n) c^{(2)} c^{(0)}(n)-W^{(1)} c^{(1)} c^{(\hat{2})}(n) c^{(0)}(n) \\
& \left.-W^{(2)} c^{(\hat{1})}(n) \grave{c}^{(2)} c^{(0)}(n)\right]
\end{aligned}
$$

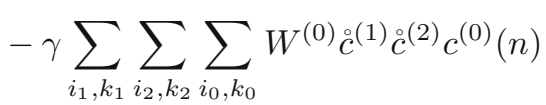

$$
\begin{aligned}
& -\gamma \frac{\delta}{2}\left\|c^{(0)}(n)\right\|^{2}+\frac{1}{2}\left\|\begin{array}{l}
c^{(1)}(n) \\
c^{(2)}(n)
\end{array}\right\|^{2} \\
& \left.+\gamma \bar{\lambda}_{n} \frac{\delta}{2}\left\|\begin{array}{l}
c^{(\hat{1})}(n) \\
c^{(\hat{2})}(n)
\end{array}\right\|^{2}+\gamma \frac{\delta}{2} \bar{\lambda}_{n}^{2}\right\}
\end{aligned}
$$

1.d. For

$$
\bar{w}_{n}=\arg \min _{w \in W}\left\{\frac{1}{2}\left\|w-w_{n}\right\|^{2}-\gamma \mathfrak{L}_{\delta}\left(x_{n}, v_{n}, w, \bar{\lambda}_{n}\right)\right\},
$$

we have

$$
\begin{aligned}
& \bar{w}_{n}=\arg \min _{w \in W}\left\{\left(\frac{1}{2}+\gamma \bar{\lambda}_{n} \frac{\delta}{2}\right)\left\|\begin{array}{l}
c^{(\hat{1})} \\
c^{(\hat{2})}
\end{array}\right\|^{2}\right. \\
& -\left(\begin{array}{l}
c^{(\hat{1})} \\
c^{(\hat{2})}
\end{array}\right)^{T}\left(\begin{array}{l}
c^{(\hat{1})}(n) \\
c^{(\hat{2})}(n)
\end{array}\right) \\
& -\gamma \bar{\lambda}_{n} \sum_{i_{1}, k_{1}} \sum_{i_{2}, k_{2}} \sum_{i_{0}, k_{0}}\left[W^{(1)} \stackrel{\circ}{(1)}^{(\hat{2})} c^{(0)}(n)\right. \\
& +W^{(2)} c^{(\hat{1}) \stackrel{(2)}{c}} c^{(0)}(n)-W^{(1)} c^{(1)}(n) c^{(\hat{2})} c^{(0)}(n) \\
& \left.-W^{(2)} c^{(\hat{1})} c^{(2)}(n) c^{(0)}(n)\right]
\end{aligned}
$$

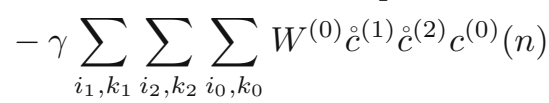

$$
\begin{aligned}
& -\gamma \frac{\delta}{2}\left\|c^{(0)}(n)\right\|^{2}+\frac{1}{2}\left\|\begin{array}{c}
c^{(\hat{1})}(n) \\
c^{(\hat{2})}(n)
\end{array}\right\|^{2}
\end{aligned}
$$

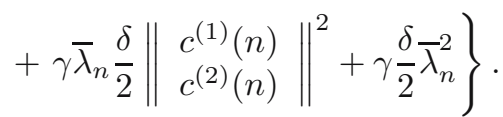

\section{Second half-step:}

2.a. For

$\lambda_{n+1}=\arg \min _{\lambda \geq 0}\left\{\frac{1}{2}\left(\lambda-\lambda_{n}\right)^{2}-\gamma \mathfrak{L}_{\delta}\left(\bar{x}_{n}, \bar{v}_{n}, \bar{w}_{n}, \lambda\right)\right\}$, 
we have

$$
\begin{aligned}
\lambda_{n+1}= & {\left[\lambda_{n}+\gamma \sum_{i_{1}, k_{1}} \sum_{i_{2}, k_{2}} \sum_{i_{0}, k_{0}}\left(W^{(1)} c^{(1)} \bar{c}^{(\hat{2})}(n) \bar{c}^{(0)}(n)\right.\right.} \\
& +W^{(2)} \bar{c}^{(\hat{1})}(n) \stackrel{c^{(2)}}{\bar{c}^{(0)}(n)} \\
& -W^{(1)} \bar{c}^{(1)}(n) \bar{c}^{(\hat{2})}(n) \bar{c}^{(0)}(n) \\
& \left.-W^{(2)} \bar{c}^{(\hat{1})}(n) \bar{c}^{(2)}(n) \bar{c}^{(0)}(n)\right) \\
& \left.-\gamma \frac{\delta}{2}\left(\left\|\bar{c}^{(1)}(n)\right\|^{(2)}(n)+\left\|\begin{array}{c}
\bar{c}^{(\hat{1})}(n) \\
\bar{c}^{(\hat{2})}(n)
\end{array}\right\|^{2}\right)\right]^{+} \\
& \times \frac{1}{1+\gamma \delta} .
\end{aligned}
$$

2.b. For

$$
x_{n+1}=\arg \min _{x \in X}\left\{\frac{1}{2}\left\|x-x_{n}\right\|^{2}+\gamma \mathfrak{L}_{\delta}\left(x, \bar{v}_{n}, \bar{w}_{n}, \bar{\lambda}_{n}\right)\right\},
$$

we obtain

$$
\begin{aligned}
& x_{n+1} \\
& =\arg \min _{x \in X}\left\{\left(\frac{1}{2}+\gamma \frac{\delta}{2}\right)\left\|c^{(0)}\right\|^{2}\right.
\end{aligned}
$$

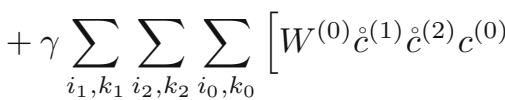

$$
\begin{aligned}
& +\bar{\lambda}_{n}\left(W^{(1)} c^{(1)} \bar{c}^{(\hat{2})}(n) c^{(0)}\right. \\
& +W^{(2)} \bar{c}^{(\hat{1})}(n) \stackrel{\circ}{(2)} c^{(0)}-W^{(1)} \bar{c}^{(1)}(n) \bar{c}^{(\hat{2})}(n) c^{(0)} \\
& \left.\left.-W^{(2)} \bar{c}^{(\hat{1})}(n) \bar{c}^{(2)}(n) c^{(0)}\right)\right] \\
& -c^{(0) T}(n) c^{(0)}+\frac{1}{2}\left\|c^{(0)}(n)\right\|^{2} \\
& -\gamma \bar{\lambda}_{n} \frac{\delta}{2}\left(\left\|\begin{array}{l}
\bar{c}^{(1)}(n) \\
\bar{c}^{(2)}(n)
\end{array}\right\|^{2}+\left\|\begin{array}{l}
\bar{c}^{(\hat{1})}(n) \\
\bar{c}^{(\hat{2})}(n)
\end{array}\right\|^{2}\right) \\
& \left.-\gamma \frac{\delta}{2} \bar{\lambda}_{n}^{2}\right\}
\end{aligned}
$$

2.c. For

$$
v_{n+1}=\arg \min _{v \in V}\left\{\frac{1}{2}\left\|v-v_{n}\right\|^{2}-\gamma \mathfrak{L}_{\delta}\left(\bar{x}_{n}, v, \bar{w}_{n}, \bar{\lambda}_{n}\right)\right\},
$$

we get

$$
\begin{aligned}
& v_{n+1} \\
& =\arg \min _{v \in V}\left\{\left(\frac{1}{2}+\gamma \bar{\lambda}_{n} \frac{\delta}{2}\right)\left\|\begin{array}{c}
c^{(1)} \\
c^{(2)}
\end{array}\right\|^{2}\right.
\end{aligned}
$$

$$
\begin{aligned}
& -\left(\begin{array}{l}
c^{(1)} \\
c^{(2)}
\end{array}\right)^{T}\left(\begin{array}{l}
c^{(1)}(n) \\
c^{(2)}(n)
\end{array}\right) \\
& +\gamma \bar{\lambda}_{n} \sum_{i_{1}, k_{1}} \sum_{i_{2}, k_{2}} \sum_{i_{0}, k_{0}}\left[W^{(1)} c^{(1)} \bar{c}^{(\hat{2})}(n) \bar{c}^{(0)}(n)\right. \\
& +W^{(2)} \bar{c}^{(\hat{1})}(n) c^{(2)} \bar{c}^{(0)}(n)-W^{(1)} c^{(1)} \bar{c}^{(\hat{2})}(n) \bar{c}^{(0)}(n) \\
& \left.-W^{(2)} \bar{c}^{(\hat{1})}(n) \grave{c}^{(2)} \bar{c}^{(0)}(n)\right]
\end{aligned}
$$

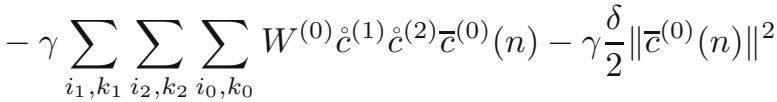

$$
\begin{aligned}
& \left.+\frac{1}{2}\left\|\begin{array}{l}
c^{(1)}(n) \\
c^{(2)}(n)
\end{array}\right\|^{2}+\gamma \bar{\lambda}_{n} \frac{\delta}{2}\left\|\begin{array}{l}
\bar{c}^{(\hat{1})}(n) \\
\bar{c}^{(\hat{2})}(n)
\end{array}\right\|^{2}+\gamma \frac{\delta}{2} \bar{\lambda}_{n}^{2}\right\} .
\end{aligned}
$$

2.d. For

$w_{n+1}=\arg \min _{w \in W}\left\{\frac{1}{2}\left\|w-w_{n}\right\|^{2}-\gamma \mathfrak{L}_{\delta}\left(\bar{x}_{n}, \bar{v}_{n}, w, \bar{\lambda}_{n}\right)\right\}$,

we have

$$
\begin{aligned}
& w_{n+1}=\arg \min _{w \in W}\left\{\left(\frac{1}{2}+\gamma \bar{\lambda}_{n} \frac{\delta}{2}\right)\left\|\begin{array}{l}
c^{(\hat{1})} \\
c^{(\hat{2})}
\end{array}\right\|^{2}\right. \\
& -\left(\begin{array}{c}
c^{(\hat{1})} \\
c^{(\hat{2})}
\end{array}\right)^{T}\left(\begin{array}{l}
c^{(\hat{1})}(n) \\
c^{(\hat{2})}(n)
\end{array}\right) \\
& -\gamma \bar{\lambda}_{n} \sum_{i_{1}, k_{1}} \sum_{i_{2}, k_{2}} \sum_{i_{0}, k_{0}}\left[W^{(1)} c^{(1)} c^{(\hat{2})} \bar{c}^{(0)}(n)\right. \\
& +W^{(2)} c_{\left(i_{1}, k_{1}\right)}^{(\hat{1})} \stackrel{\stackrel{(2)}{ }}{ }^{(0)}(n) \\
& -W^{(1)} \bar{c}^{(1)}(n) c^{(\hat{2})} \bar{c}^{(0)}(n) \\
& \text { - } \left.W^{(2)} c^{(\hat{1})} \bar{c}^{(2)}(n) \bar{c}^{(0)}(n)\right] \\
& -\gamma \sum_{i_{1}, k_{1}} \sum_{i_{2}, k_{2}} \sum_{i_{0}, k_{0}} W^{(0)} \stackrel{\circ}{c}^{(1)} \stackrel{c}{(2)}^{(0)}(n) \\
& -\gamma \frac{\delta}{2}\left\|\bar{c}^{(0)}(n)\right\|^{2}+\frac{1}{2}\left\|\begin{array}{l}
c^{(\hat{1})}(n) \\
c^{(\hat{2})}(n)
\end{array}\right\|^{2} \\
& \left.+\gamma \bar{\lambda}_{n} \frac{\delta}{2}\left\|\begin{array}{l}
\bar{c}^{(1)}(n) \\
\bar{c}^{(2)}(n)
\end{array}\right\|^{2}+\gamma \frac{\delta}{2} \bar{\lambda}_{n}^{2}\right\} .
\end{aligned}
$$

4.5. Quadratic programming solver. Let us restrict the expression (3) for the admissible set and the stationary case with the following constraint.

Each matrix $d_{\left(i_{l} \mid k_{l}\right)}^{(l)}$ represents a stationary mixed-strategy that belongs to the simplex

$$
S^{\left(N_{l} M_{l}\right)}:=\left\{\begin{array}{l}
d_{\left(i_{l} \mid k_{l}\right)}^{(l)} \in \mathbb{R}^{N_{l} M_{l}} \text { for } d_{\left(i_{l} \mid k_{l}\right)}^{(l)} \geq 0 \\
\text { where } \sum_{k_{l}=1}^{M_{l}} d_{\left(i_{l} \mid k_{l}\right)}^{(l)}=1 .
\end{array}\right.
$$


A necessary and sufficient condition for $d_{\left(i_{l} \mid k_{l}\right)}^{(l)}$ to be a Stackelberg/Nash equilibrium point is the solution of the following QPP (quadratic programming problem):

$$
\mathbf{J}^{l}\left(d_{\left(i_{0}, k_{0}\right)}^{0}, d_{\left(i_{1}, k_{1}\right)}^{1}, \ldots, d_{\left(i_{\mathcal{N}}, k_{\mathcal{N}}\right)}^{\mathcal{N}}\right) \rightarrow \min _{d_{\left(i_{l} \mid k_{l}\right)}^{(l)}}
$$

subject to

$$
d_{\left(i_{l} \mid k_{l}\right)}^{(l)} \in S^{\left(N_{l} M_{l}\right)} .
$$

We consider the problem

$$
\begin{gathered}
\frac{1}{2} X^{\top} H X+C^{\top} X \rightarrow \min _{X}, \\
A X \leq b, \quad A X=b,
\end{gathered}
$$

where $0 \leq X \leq 1, A^{l} \in \mathbb{R}^{N_{l}+1 \times\left(N_{l} M_{l}\right)}$ and $b^{l} \in \mathbb{R}^{N_{l}+1}$.

The vector $C^{T}=C_{(N \mid M)}$ is defined as

$$
\begin{aligned}
C_{(1 \mid 1)} & =W_{(1 \mid 1)}^{l}, \\
C_{(2 \mid 1)} & =W_{(2 \mid 1)}^{l}, \\
& \vdots \\
C_{(N \mid 1)} & =W_{(N \mid 1)}^{l}, \\
& \vdots \\
C_{\left(1 \mid M_{l}\right)} & =W_{\left(1 \mid M_{l}\right)}^{l}, \\
& \vdots \\
C_{\left(N_{l} \mid M_{l}\right)} & =W_{\left(N_{l} \mid M_{l}\right)}^{l}
\end{aligned}
$$

that is, $C_{(N \mid M)}$ is equal to $W_{\left(i_{1}, k_{1}, \ldots, i_{\mathcal{N}}, k_{\mathcal{N}}\right)}^{l}$ ordered by columns.

The vector $b^{l}$ is defined as

$$
b^{l}=\underset{N_{l} \text { times }}{(0, \ldots, 0,1)^{T}} \in \mathbb{R}^{N_{l}+1} .
$$

We will construct the matrix $A^{l} \in \mathbb{R}^{N_{l} \times\left(N_{l} M_{l}\right)}$ using the ergodicity constraints defined in (5),

$$
0=\sum_{k_{l}=1}^{M_{l}}\left(\sum_{i_{l}=1}^{N_{l}} \pi_{\left(i_{l} j_{l} \mid k_{l}\right)} c_{\left(i_{l} \mid k_{l}\right)}^{(l)}-c_{\left(j_{l} \mid k_{l}\right)}^{(l)}\right) .
$$

Then, we have

$$
\begin{gathered}
j_{l}=1 \sum_{k_{l}=1}^{M_{l}}\left(\sum_{i_{l}=1}^{N_{l}} \pi_{\left(i_{l} j_{l} \mid k_{l}\right)} c_{\left(i_{l} \mid k_{l}\right)}^{(l)}-c_{\left(1 \mid k_{l}\right)}^{(l)}\right)=0, \\
j_{l}=2 \sum_{k_{l}=1}^{M_{l}}\left(\sum_{i_{l}=1}^{N_{l}} \pi_{\left(i_{l} j_{l} \mid k_{l}\right)} c_{\left(i_{l} \mid k_{l}\right)}^{(l)}-c_{\left(2 \mid k_{l}\right)}^{(l)}\right)=0, \\
\vdots \\
j_{l}=N_{l} \sum_{k_{l}=1}^{M_{l}}\left(\sum_{i_{l}=1}^{N_{l}} \pi_{\left(i_{l} j_{l} \mid k_{l}\right)} c_{\left(i_{l} \mid k_{l}\right)}^{(l)}-c_{\left(N_{l} \mid k_{l}\right)}^{(l)}\right)=0 .
\end{gathered}
$$

Developing the formulas of (31) for $\mathcal{N}$ players and multiplying by $c_{\left(i_{l} \mid k_{l}\right)}^{(l)}$ for each component (31), we have

$$
\begin{aligned}
B L^{(l)} & =\left[\pi_{\left(j_{l}, i_{l} \mid k_{l}\right)}^{(l)}-\delta_{\left(j_{l}, i_{l}\right)}\right]_{j_{l}=\overline{1, N_{l}} i_{l}=\overline{1, N_{l}}} \\
& =\left[\begin{array}{ccc}
\pi_{(1,1 \mid 1)}^{(l)}-1 & \ldots & \pi_{\left(N_{l}, 1 \mid M_{l}\right)}^{(l)} \\
\ldots & \ldots & \ldots \\
\pi_{\left(N_{l}, 1 \mid 1\right)}^{(l)} & \ldots & \pi_{\left(N_{l}, N_{l} \mid M_{l}\right)}^{(l)}-1
\end{array}\right],
\end{aligned}
$$

where $\delta_{\left(j_{l}, i_{l}\right)}$ is Kronecker's delta. Then $A_{\text {eq }} \in$ $\mathbb{R}^{\left(\sum_{l=1}^{\mathcal{N}} N_{l}+(\mathcal{N})\right) \times\left(\sum_{l=1}^{\mathcal{N}} N_{l} \times M_{l}\right)}$ is defined as follows:

$$
A_{\mathrm{eq}}=\left[\begin{array}{cccc}
B L^{(1)} & 0 & \ldots & 0 \\
0 & B L^{(2)} & \ldots & 0 \\
\ldots \ldots \ldots & \ldots \ldots & \ldots \ldots \ldots \ldots \\
0 & 0 & \ldots & B L^{\left(M_{l}\right)} \\
{[1]} & 0 & \ldots & 0 \\
0 & {[1]} & \ldots & 0 \\
0 & 0 & \ldots & 0 \\
0 & 0 & 0 & {[1]}
\end{array}\right]
$$

where

$$
[1]=(1, \ldots, 1)^{\top} \in \mathbb{R}^{\left(N_{l}\right)}
$$

and

$$
b_{\text {eq }} \in \mathbb{R}^{\left(\sum_{l=1}^{\mathcal{N}} N_{l}+(\mathcal{N})\right)}
$$

is

$$
b_{\mathrm{eq}}=\left(\begin{array}{llllll}
0 & \ldots & 0 & 1 & \ldots & 1
\end{array}\right)^{\top} .
$$

\section{Convergence analysis}

5.1. Auxiliary results. Let us prove the following auxiliary results.

Lemma 2. Let $f(z)$ be a convex function defined on a convex set $Z$. If $z^{*}$ is a minimizer of function

$$
\varphi(z)=\frac{1}{2}\|z-x\|^{2}+\alpha f(z)
$$

on $Z$ with fixed $x$, then $f(z)$ satisfies the inequality

$$
\begin{aligned}
\frac{1}{2}\left\|z^{*}-x\right\|^{2} & +\alpha f\left(z^{*}\right) \\
& \leq \frac{1}{2}\|z-x\|^{2}+\alpha f(z)-\frac{1}{2}\left\|z-z^{*}\right\|^{2} .
\end{aligned}
$$

Proof. By the necessary condition for a minimum at $z^{*}$,

$$
\left\langle z^{*}-x+\alpha \nabla f\left(z^{*}\right), z-z^{*}\right\rangle \geq 0,
$$

and the convexity property of $f(z)$, expressed as

$$
f(z) \geq f\left(z^{*}\right)+\left\langle\nabla f\left(z^{*}\right), z-z^{*}\right\rangle,
$$

we derive

$$
\begin{aligned}
0 & \leq\left\langle z^{*}-x+\alpha \nabla f\left(z^{*}\right), z-z^{*}\right\rangle \\
& =\left\langle z^{*}-x, z-z^{*}\right\rangle+\alpha\left[f(z)-f\left(z^{*}\right)\right] .
\end{aligned}
$$


Using this inequality in the identity

$$
\begin{aligned}
\frac{1}{2} \| z- & x \|^{2} \\
& =\frac{1}{2}\left\|z-z^{*}\right\|^{2}+\left\langle z^{*}-x, z-z^{*}\right\rangle+\frac{1}{2}\left\|z^{*}-x\right\|^{2},
\end{aligned}
$$

we get (33). The lemma is proven.

Lemma 3. If all partial derivatives of $\tilde{\mathcal{L}}_{\delta}(\tilde{x}, \tilde{y})$ satisfy the Lipschitz condition with positive constant $C_{0}$, then the following Lipschitz-type condition holds:

$$
\begin{aligned}
& \|\left[\Psi_{\delta}(\tilde{w}+h, \tilde{v}+g)-\Psi_{\delta}(\tilde{w}, \tilde{v}+g)\right] \\
& \quad-\left[\Psi_{\delta}(\tilde{w}+h, \tilde{v})-\Psi_{\delta}(\tilde{w}, \tilde{v})\right]\left\|\leq C_{0}\right\| h\|\| g \|
\end{aligned}
$$

valid for any $\tilde{w}, h, \tilde{v}, g \in \tilde{X} \times \tilde{Y}$.

Proof. By Lagrange's formula

$$
f(x+h)-f(x)=\int_{0}^{1}\langle\nabla f(x+t h), h\rangle \mathrm{d} t,
$$

we have

$$
\begin{aligned}
& \|\left[\Psi_{\delta}(\tilde{w}+h, \tilde{v}+g)-\Psi_{\delta}(\tilde{w}, \tilde{v}+g)\right] \\
& -\left[\Psi_{\delta}(\tilde{w}+h, \tilde{v})-\Psi_{\delta}(\tilde{w}, \tilde{v})\right] \| \\
= & \left\|\int_{0}^{1}\left\langle\nabla \Psi_{\delta}(\tilde{w}+t h, \tilde{v}+g)-\nabla \Psi_{\delta}(\tilde{w}+t h, \tilde{v}), h\right\rangle \mathrm{d} t\right\| \\
\leq & \int_{0}^{1}\left|\left\langle\nabla \Psi_{\delta}(\tilde{w}+t h, \tilde{v}+g)-\nabla \Psi_{\delta}(\tilde{w}+t h, \tilde{v}), h\right\rangle\right| \mathrm{d} t \\
\leq & \int_{0}^{1} C_{0}\|h\|\|g\| \mathrm{d} t \leq C_{0}\|h\|\|k\|,
\end{aligned}
$$

which proves the lemma.

5.2. Main convergence theorem. The following theorem presents the convergence conditions of (17)- (19) and gives an estimate of its rate of convergence.

Theorem 1. Assume that the problem (16) has a solution. Let $\tilde{\mathcal{L}}_{\delta}(\tilde{x}, \tilde{y})$ be differentiable in $\tilde{x}$ and $\tilde{y}$, whose partial derivative with respect to $\tilde{y}$ satisfies the Lipschitz condition with positive constant $C$. Then, for any $\delta \in(0,1)$, there exists a small enough

$$
\gamma_{0}=\gamma_{0}(\delta)<C:=\min \left\{\frac{1}{\sqrt{2} C_{0}}, \frac{1+\sqrt{1+2 C_{0}^{2}}}{2 C_{0}^{2}}\right\}
$$

such that, for any $0<\gamma \leq \gamma_{0}$, the sequence $\left\{\tilde{v}_{n}\right\}$, generated by the equivalent extraproximal procedure (18)-(20), monotonically converges in norm with a geometric progression rate $q \in(0,1)$ to one of the equilibrium points $\widetilde{v}^{*}$, i.e.

$$
\left\|\widetilde{v}_{n}-\widetilde{v}^{*}\right\|^{2} \leq q^{n}\left\|\widetilde{v}_{0}-\widetilde{v}^{*}\right\|^{2},
$$

where

$$
q=1+\frac{4(\delta \gamma)^{2}}{1+2 \delta \gamma-2 \gamma^{2} C^{2}}-2 \delta \gamma
$$

Proof. Taking in (33) $\alpha=\gamma$ and

$$
\begin{gathered}
z=\tilde{w}, \quad x=\tilde{v}_{n}, \quad z^{*}=\hat{v}_{n}, \\
f(z)=\Psi_{\delta}\left(\tilde{w}, \tilde{v}_{n}\right), \quad f\left(z^{*}\right)=\Psi_{\delta}\left(\hat{v}_{n}, \tilde{v}_{n}\right),
\end{gathered}
$$

we obtain

$$
\begin{aligned}
& \frac{1}{2}\left\|\hat{v}_{n}-\widetilde{v}_{n}\right\|^{2}+\gamma \Psi_{\delta}\left(\hat{v}_{n}, \tilde{v}_{n}\right) \\
& \quad \leq \frac{1}{2}\left\|\tilde{w}-\tilde{v}_{n}\right\|^{2}+\gamma \Psi_{\delta}\left(\tilde{w}, \tilde{v}_{n}\right)-\frac{1}{2}\left\|\tilde{w}-\hat{v}_{n}\right\|^{2} .
\end{aligned}
$$

Again setting in (33) $\alpha=\gamma$ and

$$
\begin{gathered}
z=\tilde{w}, \quad x=\tilde{v}_{n}, \quad z^{*}=\tilde{v}_{n+1}, \\
f(z)=\Psi_{\delta}\left(\tilde{w}, \hat{v}_{n}\right), \quad f\left(z^{*}\right)=\Psi_{\delta}\left(\tilde{v}_{n+1}, \hat{v}_{n}\right),
\end{gathered}
$$

we get

$$
\begin{aligned}
& \frac{1}{2}\left\|\tilde{v}_{n+1}-\tilde{v}_{n}\right\|^{2}+\gamma \Psi_{\delta}\left(\tilde{v}_{n+1}, \hat{v}_{n}\right) \\
& \quad \leq \frac{1}{2}\left\|\tilde{w}-\tilde{v}_{n}\right\|^{2}+\gamma \Psi_{\delta}\left(\tilde{w}, \hat{v}_{n}\right)-\frac{1}{2}\left\|\tilde{w}-\tilde{v}_{n+1}\right\|^{2} .
\end{aligned}
$$

Selecting $\tilde{w}=\tilde{v}_{n+1}$ in (37) and $\tilde{w}=\hat{v}_{n}$ in 38, we obtain

$$
\begin{gathered}
\frac{1}{2}\left\|\hat{v}_{n}-\tilde{v}_{n}\right\|^{2}+\gamma \Psi_{\delta}\left(\hat{v}_{n}, \tilde{v}_{n}\right) \\
\leq \frac{1}{2}\left\|\tilde{v}_{n+1}-\tilde{v}_{n}\right\|^{2}+\gamma \Psi_{\delta}\left(\tilde{v}_{n+1}, \tilde{v}_{n}\right) \\
\quad-\frac{1}{2}\left\|\tilde{v}_{n+1}-\hat{v}_{n}\right\|^{2}, \\
\frac{1}{2}\left\|\tilde{v}_{n+1}-\tilde{v}_{n}\right\|^{2}+\gamma \Psi_{\delta}\left(\tilde{v}_{n+1}, \hat{v}_{n}\right) \\
\leq \frac{1}{2}\left\|\hat{v}_{n}-\tilde{v}_{n}\right\|^{2}+\gamma \Psi_{\delta}\left(\hat{v}_{n}, \hat{v}_{n}\right)-\frac{1}{2}\left\|\hat{v}_{n}-\tilde{v}_{n+1}\right\|^{2} .
\end{gathered}
$$

Adding (39) with (40) and using (34) for

$$
\begin{gathered}
\tilde{w}+h=\tilde{v}_{n+1}, \quad \tilde{w}=\hat{v}_{n}, \quad \tilde{v}+g=\tilde{v}_{n}, \quad \tilde{v}=\hat{v}_{n}, \\
h=\tilde{v}_{n+1}-\hat{v}_{n}, \quad g=\tilde{v}_{n}-\hat{v}_{n},
\end{gathered}
$$

we finally conclude that

$$
\begin{aligned}
\left\|\tilde{v}_{n+1}-\hat{v}_{n}\right\|^{2} \leq & \gamma\left[\Psi_{\delta}\left(\tilde{v}_{n+1}, \tilde{v}_{n}\right)-\Psi_{\delta}\left(\hat{v}_{n}, \tilde{v}_{n}\right)\right] \\
& -\gamma\left[\Psi_{\delta}\left(\tilde{v}_{n+1}, \hat{v}_{n}\right)-\Psi_{\delta}\left(\hat{v}_{n}, \hat{v}_{n}\right)\right] \\
\leq & \gamma C\left\|\tilde{v}_{n+1}-\widehat{v}_{n}\right\|\left\|\tilde{v}_{n}-\hat{v}_{n}\right\|,
\end{aligned}
$$

which implies

$$
\left\|\tilde{v}_{n+1}-\hat{v}_{n}\right\| \leq \gamma C\left\|\tilde{v}_{n}-\hat{v}_{n}\right\| .
$$

Now, taking $\tilde{w}=\tilde{v}_{n+1}$ in (37) and $\tilde{w}=\tilde{v}_{\delta}^{*}$ in (38), we get

$$
\begin{aligned}
& \frac{1}{2}\left\|\hat{v}_{n}-\tilde{v}_{n}\right\|^{2}+\gamma \Psi_{\delta}\left(\hat{v}_{n}, \tilde{v}_{n}\right) \\
& \quad \leq \frac{1}{2}\left\|\tilde{v}_{n+1}-\tilde{v}_{n}\right\|^{2}+\gamma \Psi_{\delta}\left(\tilde{v}_{n+1}, \tilde{v}_{n}\right) \\
& \quad-\frac{1}{2}\left\|\tilde{v}_{n+1}-\hat{v}_{n}\right\|^{2},
\end{aligned}
$$




$$
\begin{aligned}
& \frac{1}{2}\left\|\tilde{v}_{n+1}-\tilde{v}_{n}\right\|^{2}+\gamma \Psi_{\delta}\left(\tilde{v}_{n+1}, \hat{v}_{n}\right) \\
& \leq \frac{1}{2}\left\|\tilde{v}_{\delta}^{*}-\tilde{v}_{n}\right\|^{2}+\gamma \Psi_{\delta}\left(\tilde{v}_{\delta}^{*}, \hat{v}_{n}\right)-\frac{1}{2}\left\|\tilde{v}_{\delta}^{*}-\tilde{v}_{n+1}\right\|^{2} .
\end{aligned}
$$

Adding these two inequalities and multiplying by two yields

$$
\begin{aligned}
& \left\|\tilde{v}_{\delta}^{*}-\tilde{v}_{n+1}\right\|^{2}+\left\|\tilde{v}_{n+1}-\hat{v}_{n}\right\|^{2}+\left\|\hat{v}_{n}-\tilde{v}_{n}\right\|^{2} \\
& \quad-2 \gamma \Psi_{\delta}\left(\tilde{v}_{\delta}^{*}, \hat{v}_{n}\right)+2 \gamma\left[\Psi_{\delta}\left(\tilde{v}_{n+1}, \hat{v}_{n}\right)\right. \\
& \left.\quad+\Psi_{\delta}\left(\hat{v}_{n}, \tilde{v}_{n}\right)-\Psi_{\delta}\left(\tilde{v}_{n+1}, \tilde{v}_{n}\right)\right] \leq\left\|\tilde{v}_{\delta}^{*}-\tilde{v}_{n}\right\|^{2} .
\end{aligned}
$$

Adding and subtracting the term $\Psi_{\delta}\left(\hat{v}_{n}, \hat{v}_{n}\right)$, we have

$$
\begin{aligned}
& \left\|\tilde{v}_{\delta}^{*}-\tilde{v}_{n+1}\right\|^{2}+\left\|\tilde{v}_{n+1}-\hat{v}_{n}\right\|^{2}+\left\|\hat{v}_{n}-\tilde{v}_{n}\right\|^{2} \\
& +2 \gamma\left[\Psi_{\delta}\left(\hat{v}_{n}, \hat{v}_{n}\right)-\Psi_{\delta}\left(\tilde{v}_{\delta}^{*}, \hat{v}_{n}\right)\right]+2 \gamma\left[\Psi_{\delta}\left(\tilde{v}_{n+1}, \hat{v}_{n}\right)\right. \\
& \left.\quad-\Psi_{\delta}\left(\hat{v}_{n}, \hat{v}_{n}\right)+\Psi_{\delta}\left(\hat{v}_{n}, \tilde{v}_{n}\right)-\Psi_{\delta}\left(\tilde{v}_{n+1}, \tilde{v}_{n}\right)\right] \\
& \quad \leq\left\|\tilde{v}_{\delta}^{*}-\tilde{v}_{n}\right\|^{2} .
\end{aligned}
$$

Using (34) with $\tilde{w}+h=\tilde{v}_{n+1}, \tilde{w}=\hat{v}_{n}, \tilde{v}+k=\tilde{v}_{n}$ and $\tilde{v}=\hat{v}_{n}$, we have $h=\tilde{v}_{n+1}-\hat{v}_{n}$ and $k=\tilde{v}_{n}-\hat{v}_{n}$, and the inequality above becomes

$$
\begin{aligned}
& \left\|\tilde{v}_{\delta}^{*}-\tilde{v}_{n+1}\right\|^{2}+\left\|\tilde{v}_{n+1}-\hat{v}_{n}\right\|^{2}+\left\|\hat{v}_{n}-\tilde{v}_{n}\right\|^{2} \\
& \quad+2 \gamma\left[\Psi_{\delta}\left(\hat{v}_{n}, \hat{v}_{n}\right)-\Psi_{\delta}\left(\tilde{v}_{\delta}^{*}, \hat{v}_{n}\right)\right] \\
& \quad-2 \gamma C\left\|\tilde{v}_{n+1}-\hat{v}_{n}\right\|\left\|\tilde{v}_{n}-\hat{v}_{n}\right\| \leq\left\|\tilde{v}_{\delta}^{*}-\tilde{v}_{n}\right\|^{2}
\end{aligned}
$$

Applying (41) to the last term on the left-hand side and in view of the strict convexity property of $\Psi_{\delta}$ given by

$$
\Psi_{\delta}\left(\hat{v}_{n}, \hat{v}_{n}\right)-\Psi_{\delta}\left(\tilde{v}_{\delta}^{*}, \hat{v}_{n}\right) \geq \delta\left\|\hat{v}_{n}-\tilde{v}_{\delta}^{*}\right\|^{2},
$$

we get

$$
\begin{aligned}
& \left\|\tilde{v}_{\delta}^{*}-\tilde{v}_{n+1}\right\|^{2}+\left\|\tilde{v}_{n+1}-\hat{v}_{n}\right\|^{2}+2 \gamma \delta\left\|\hat{v}_{n}-\tilde{v}_{\delta}^{*}\right\|^{2} \\
& \quad+\left(1-2 \gamma^{2} C^{2}\right)\left\|\tilde{v}_{n}-\hat{v}_{n}\right\|^{2} \leq\left\|\tilde{v}_{\delta}^{*}-\tilde{v}_{n}\right\|^{2} .
\end{aligned}
$$

Applying the identity $2\langle a-c, c-b\rangle=\|a-b\|^{2}-$ $\|a-c\|^{2}-\|c-b\|^{2}$ with $a=\hat{v}_{n}, b=\tilde{v}_{\delta}^{*}$ and $c=\tilde{v}_{n}$ to the left-hand side of the last inequality, we have

$$
\begin{aligned}
& \left\|\tilde{v}_{\delta}^{*}-\tilde{v}_{n+1}\right\|^{2}+\left\|\tilde{v}_{n+1}-\hat{v}_{n}\right\|^{2}+\left(1-2 \gamma^{2} C^{2}\right)\left\|\tilde{v}_{n}-\hat{v}_{n}\right\|^{2} \\
& +2 \gamma \delta\left[2\left\langle\hat{v}_{n}-\tilde{v}_{n}, \tilde{v}_{n}-\tilde{v}_{\delta}^{*}\right\rangle+\left\|\tilde{v}_{n}-\hat{v}_{n}\right\|^{2}+\left\|\tilde{v}_{n}-\tilde{v}_{\delta}^{*}\right\|^{2}\right. \\
& =\left\|\tilde{v}_{\delta}^{*}-\tilde{v}_{n+1}\right\|^{2}+\left\|\tilde{v}_{n+1}-\hat{v}_{n}\right\|^{2} \\
& \quad+\left(1+2 \gamma \delta-2 \gamma^{2} C^{2}\right)\left\|\tilde{v}_{n}-\hat{v}_{n}\right\|^{2} \\
& \quad+4 \gamma \delta\left\langle\hat{v}_{n}-\tilde{v}_{n}, \tilde{v}_{n}-\tilde{v}_{\delta}^{*}\right\rangle \\
& \quad+2 \gamma \delta\left\|\tilde{v}_{n}-\tilde{v}_{\delta}^{*}\right\|^{2} \leq\left\|\tilde{v}_{\delta}^{*}-\tilde{v}_{n}\right\|^{2} .
\end{aligned}
$$

Defining $d=1+2 \gamma \delta-2 \gamma^{2} C^{2}$ and completing the square form of the third and fourth terms yields

$$
\begin{aligned}
& \left\|\tilde{v}_{\delta}^{*}-\tilde{v}_{n+1}\right\|^{2}+\left\|\tilde{v}_{n+1}-\hat{v}_{n}\right\|^{2}+d\left\|\tilde{v}_{n}-\hat{v}_{n}\right\|^{2} \\
& \quad+4 \gamma \delta\left\langle\hat{v}_{n}-\tilde{v}_{n}, \tilde{v}_{n}-\tilde{v}_{\delta}^{*}\right\rangle+\frac{(2 \gamma \delta)^{2}}{d}\left\|\tilde{v}_{n}-\tilde{v}_{\delta}^{*}\right\|^{2} \\
& \quad-\frac{(2 \gamma \delta)^{2}}{d}\left\|\tilde{v}_{n}-\tilde{v}_{\delta}^{*}\right\|^{2}+2 \gamma \delta\left\|\tilde{v}_{n}-\tilde{v}_{\delta}^{*}\right\|^{2} \leq\left\|\tilde{v}_{\delta}^{*}-\tilde{v}_{n}\right\|^{2}
\end{aligned}
$$

and

$$
\begin{aligned}
& \left\|\tilde{v}_{\delta}^{*}-\tilde{v}_{n+1}\right\|^{2}+\left\|\tilde{v}_{n+1}-\hat{v}_{n}\right\|^{2} \\
& +\left\|\sqrt{d}\left(\tilde{v}_{n}-\hat{v}_{n}\right)+\frac{2 \gamma \delta}{\sqrt{d}}\left(\tilde{v}_{n}-\tilde{v}_{\delta}^{*}\right)\right\|^{2} \\
& \quad \leq\left(1-2 \gamma \delta+\frac{(2 \gamma \delta)^{2}}{d}\right)\left\|\tilde{v}_{\delta}^{*}-\tilde{v}_{n}\right\|^{2}
\end{aligned}
$$

finally implying that

$$
\begin{aligned}
\left\|\tilde{v}_{\delta}^{*}-\tilde{v}_{n+1}\right\|^{2} & \leq q\left\|\tilde{v}_{\delta}^{*}-\tilde{v}_{n}\right\|^{2} \\
& \leq q^{n+1}\left\|\tilde{v}_{\delta}^{*}-\tilde{v}_{0}\right\|^{2} \underset{n \rightarrow \infty}{\longrightarrow} 0
\end{aligned}
$$

with

$$
q=1-2 \gamma \delta+\frac{(2 \gamma \delta)^{2}}{d} \in(0,1) .
$$

The theorem is proven.

\section{Numerical example}

We consider a pricing oligopoly model (Karpowicz, 2012; Nowak and Romaniuk, 2013) with a leader and two followers. For instance, let us consider AA, one of the most profitable airlines globally, which has always had the reputation of a leader and industry challenger. Let us also consider two local flying companies called AI and AII as the followers of AA on the market. AI and AII were forced to immediately start competing with international airlines for routes, getting access to airports, securing flight slots and landing rights, and attracting a new customer base.

We suppose that each consumer buys at most one ticket. It is a common practice for airlines to price discriminate, i.e., consumers coming to the market at different times have valuations inversely related to the length of time between the purchase and the flight. This is the case of business people who decide to travel at the last moment, and pay much more money for getting a ticket. On the other hand, people who plan to travel a year ahead pay significantly less money. The linear demand assumes a continuum of people between these extremes.

The dynamics of the game are as follows: (a) AA is the first mover and manages all its advantage attracting the highest value customers who pay a uniformly high price, (b) AI and AII price discriminates over the residual demand. The firms can choose to offer limited airline seats for a specific route: high, medium, low, or none at all. The market price of the seats decreases with increasing total quantity offered by the companies. If the companies decide to offer a high quantity of airline seats, the price collapses so that profits drop to zero. Figure 1 describes the states and actions corresponding to the Markov chain of the pricing problem. 


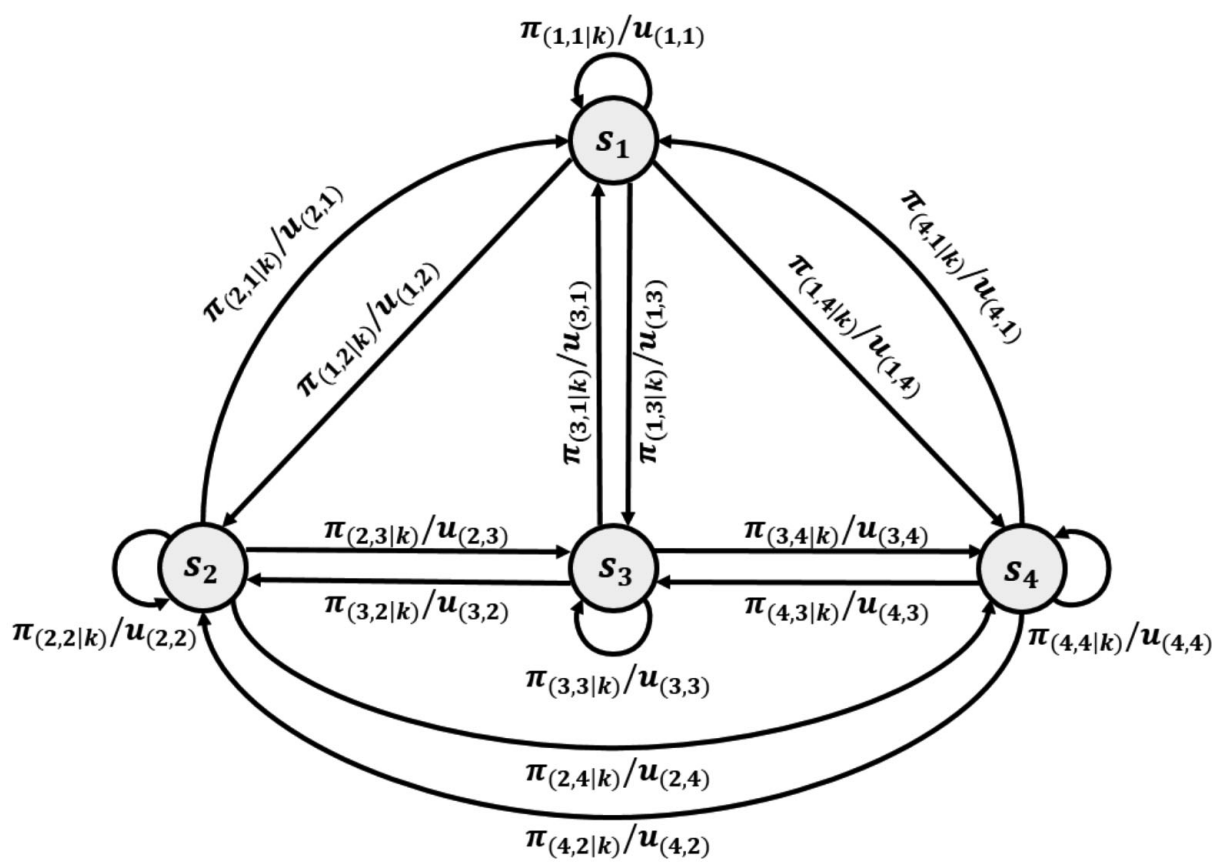

Fig. 1. Pricing Markov chain.

Following Eqn. (2), the individual utilities are as follows:

$\begin{array}{ccccc}\text { AA } & \text { high } & \text { medium } & \text { low } & \text { none } \\ \text { High } & 0 & 30 & 43 & 69 \\ \text { Medium } & 22 & 39 & 47 & 71 \\ \text { Low } & 25 & 37 & 43 & 61 \\ \text { None } & 0 & 0 & 0 & 0\end{array}$

$$
\pi_{(i, j \mid 1)}^{A I I}=\left[\begin{array}{llll}
0.2564 & 0.7202 & 0.0005 & 0.0230 \\
0.0115 & 0.3802 & 0.2628 & 0.3455 \\
0.2662 & 0.0333 & 0.3895 & 0.3111 \\
0.0513 & 0.5530 & 0.3145 & 0.0812
\end{array}\right]
$$$$
\pi_{(i, j \mid 2)}^{A I I}=\left[\begin{array}{llll}
0.0077 & 0.0217 & 0.0346 & 0.9360 \\
0.0758 & 0.3909 & 0.3933 & 0.1401 \\
0.2946 & 0.1857 & 0.1726 & 0.3470 \\
0.0611 & 0.2346 & 0.5648 & 0.1395
\end{array}\right]
$$

$\begin{array}{ccccc}\text { AI } & \text { high } & \text { medium } & \text { low } & \text { none } \\ \text { High } & 0 & 22 & 24 & 0 \\ \text { Medium } & 30 & 39 & 37 & 0 \\ \text { Low } & 43 & 47 & 43 & 0 \\ \text { None } & 59 & 41 & 55 & 0\end{array}$

$\begin{array}{ccccc}\text { AII } & \text { high } & \text { medium } & \text { low } & \text { none } \\ \text { High } & 0 & 24 & 27 & 3 \\ \text { Medium } & 28 & 39 & 39 & 5 \\ \text { Low } & 37 & 45 & 43 & 10 \\ \text { None } & 54 & 39 & 59 & 0\end{array}$

$$
\begin{aligned}
\pi_{(i, j \mid 1)}^{A A} & =\left[\begin{array}{llll}
0.0239 & 0.8486 & 0.0471 & 0.0804 \\
0.2961 & 0.3267 & 0.2228 & 0.1543 \\
0.0975 & 0.1078 & 0.6152 & 0.1794 \\
0.2028 & 0.2719 & 0.3546 & 0.1707
\end{array}\right], \\
\pi_{(i, j \mid 2)}^{A A} & =\left[\begin{array}{llll}
0.3579 & 0.0507 & 0.2801 & 0.3113 \\
0.5572 & 0.3785 & 0.0120 & 0.0523 \\
0.1751 & 0.2076 & 0.4572 & 0.1602 \\
0.0829 & 0.2980 & 0.3511 & 0.2681
\end{array}\right] .
\end{aligned}
$$

$$
\begin{gathered}
\pi_{(i, j \mid 1)}^{A I}=\left[\begin{array}{llll}
0.3288 & 0.2152 & 0.3739 & 0.0821 \\
0.1308 & 0.3639 & 0.4474 & 0.0578 \\
0.1394 & 0.1225 & 0.4843 & 0.2538 \\
0.5846 & 0.1538 & 0.0937 & 0.1679
\end{array}\right], \\
\pi_{(i, j \mid 2)}^{A I}=\left[\begin{array}{llll}
0.4525 & 0.3901 & 0.1061 & 0.0513 \\
0.0755 & 0.2378 & 0.3888 & 0.2979 \\
0.0373 & 0.1512 & 0.7654 & 0.0461 \\
0.2750 & 0.1673 & 0.3104 & 0.2474
\end{array}\right],
\end{gathered}
$$

Each company knows how the increased offer lowers airline seats price and their profits. Strategic dependence is a common practice in the global airline industry. In this case, AA does this so it can predict the strategic pricing movements of other airlines. By anticipating the predicted response of the followers, AA is able to make a strategy to become and stay successful. Given $\delta=14.3, \gamma=$ 
0.008 and applying the extraproximal method for Markov chains (see Section 4.4), we obtain $c_{(i \mid k)}$ for the leader (see Fig. 3) and $c_{(i \mid k)}$ for the followers (see Figs. 4 and 5). Figure 2 shows the convergence of the parameter $\lambda$. Then, applying (6), we have that the strategies needed to converge to a Stackelberg equilibrium are as follows:

$$
\begin{aligned}
d^{A A} & =\left[\begin{array}{ll}
0.8995 & 0.1005 \\
0.0656 & 0.9344 \\
0.2172 & 0.7828 \\
0.2827 & 0.7173
\end{array}\right], \\
d^{A I} & =\left[\begin{array}{ll}
0.1077 & 0.8923 \\
0.1311 & 0.8689 \\
0.9035 & 0.0965 \\
0.8349 & 0.1651
\end{array}\right], \\
d^{A I I} & =\left[\begin{array}{ll}
0.7331 & 0.2669 \\
0.9214 & 0.0786 \\
0.1230 & 0.8770 \\
0.8856 & 0.1144
\end{array}\right] .
\end{aligned}
$$

Air travel has become a commodity and major routes are saturated with fierce competition. Low-cost carriers have significantly influenced consumer behavior for cheap price bargains among regular travelers and increasingly among business travelers. AA has consistently been one of the most profitable airlines globally, and has always had the reputation of a leader and industry challenger fixing its strategies (42) only on the most lucrative routes. $\mathrm{AI}$, looking at the strategies of AA, decided on a fully branded product/service differentiation strategy (43) as follows: (a) to compete with AA choosing a mixed strategy $d_{(1,2)}^{l}=0,8923$ for a lucrative international route and the rest for $d_{(1,1)}^{l}=0.1077$ a domestic route seriously considered by AA, (b) to strongly compete with AA in strategy $d_{(2,1)}^{l}$ for an international route, and (c) to fully compete with AA in strategies $d_{(3,1)}^{l}$ and $d_{(4,1)}^{l}$ for the most lucrative domestic routes. AII, instead, launch a strategy (44) for local and short-haul routes to stay at the forefront of competition deciding (a) to compete with AA in strategy $d_{(1,1)}^{l}$ for a domestic route, (b) to compete partially with AA choosing a mixed strategy $d_{(2,2)}^{l}=$ 0.0786 for a lucrative international route and the rest for $d_{(2,1)}^{l}=0.9214$ a local route not seriously considered by AA, (c) to absolutely compete with AA in strategy $d_{(3,2)}^{l}$ choosing seriously only an international route, and (d) to partially compete with AA choosing a mixed strategy $d_{(4,2)}^{l}=0.1144$ for a lucrative international route and the rest for $d_{(4,1)}^{l}=0.8856$ a local route partially considered by AA.

Remark 2. Price efficiency and effectiveness are critical for an airline's ability to compete and survive. However, this does not mean that every airline should seek to offer the lowest price. Instead, it is important to offer the appropriate price for routes not considered by the leader airline.

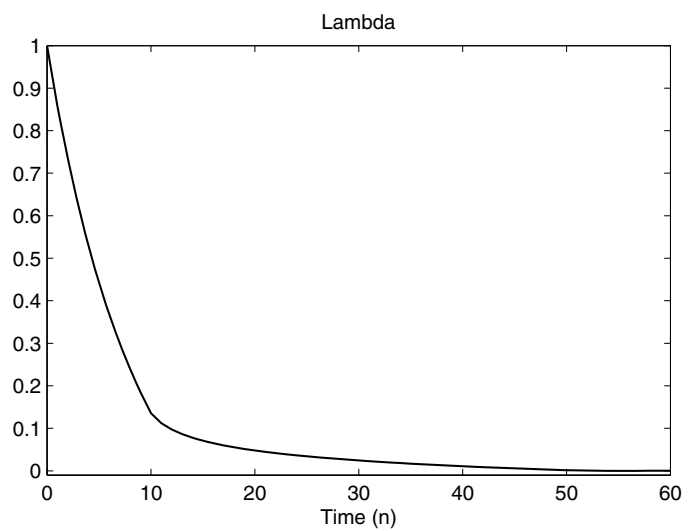

Fig. 2. Convergence of the parameter $\lambda$.

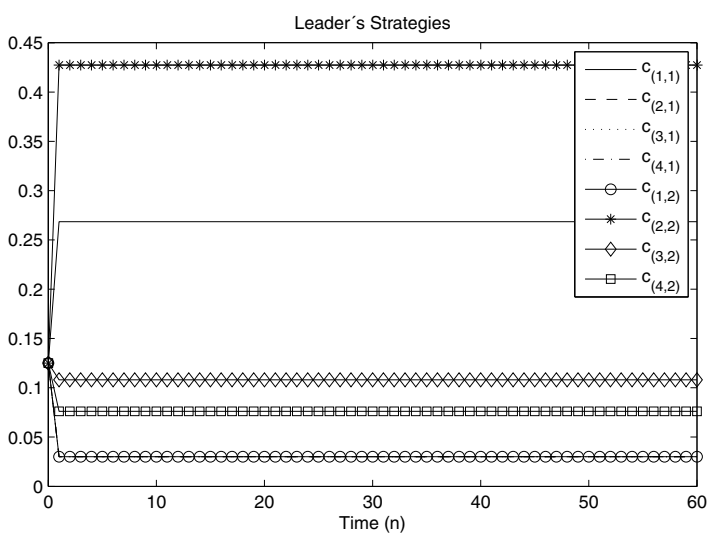

Fig. 3. Convergence of the strategies of AA.

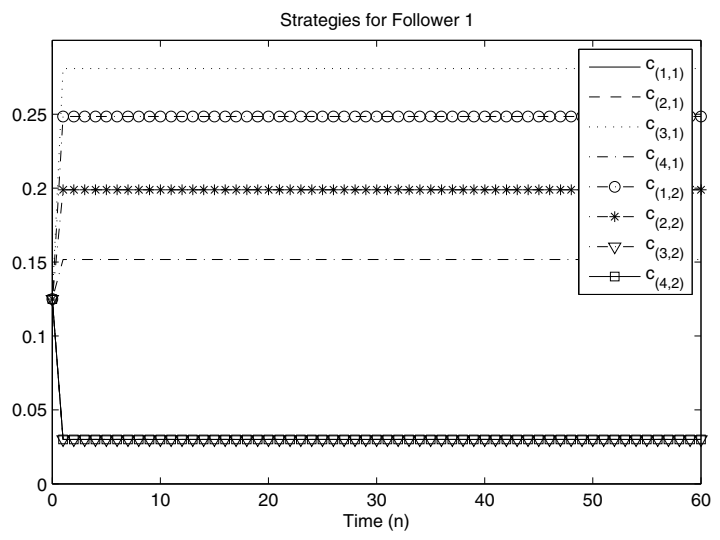

Fig. 4. Convergence of the strategies of AI. 


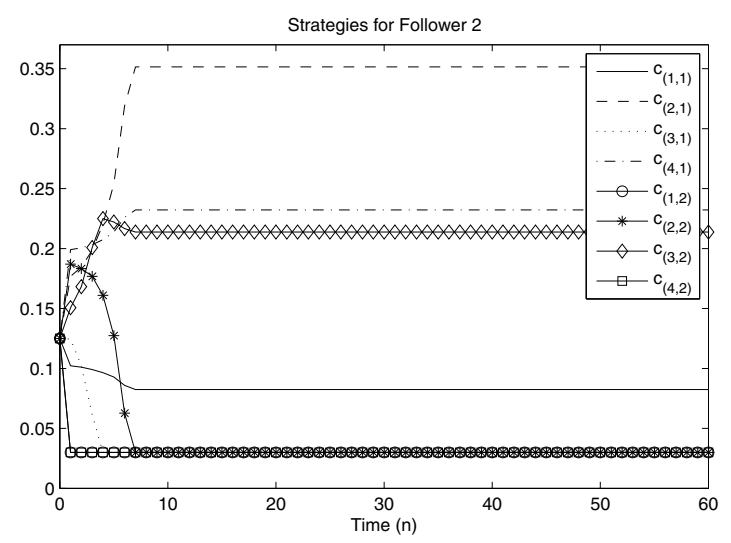

Fig. 5. Convergence of the strategies of AII.

\section{Conclusion}

The main contribution of this paper was the development of the extraproximal method for computing the Stackelberg/Nash equilibria in a class of ergodic controlled finite Markov chains games. The nonlinear programming problem was represented using an implementation of the Lagrange principle. Another important contribution was the use of the regularizing parameter, which provides strong convexity for the cost-functions and, hence, the correctness of the convergence analysis.

For solving the extraproximal method, we presented a two-step iterated procedure which involved an iterative solution of a quadratic programming problem for the solution of the Stackelberg game in terms of Markov chains. A numerical method was presented for computing the first step of the extraproximal method (parameter $\lambda$ ). The convergence of the suggested procedure to the Stackelberg/Nash equilibrium was also analyzed. It is important to note that we provided all the details needed to implement the extraproximal method in an efficient and numerically stable way for ergodic controlled finite Markov chains games.

\section{References}

Antipin, A.S. (2005). An extraproximal method for solving equilibrium programming problems and games, Computational Mathematics and Mathematical Physics 45(11): 1893-1914.

Bos, D. (1986). Public Enterprise Economics, North-Holland, Amsterdam.

Bos, D. (1991). Privatization: A Theoretical Treatment, Clarendon Press, Oxford.
Breitmoser, Y. (2012). On the endogeneity of Cournot, Bertrand, and Stackelberg competition in oligopolies, International Journal of Industrial Organization 30(1): 16-29.

Clempner, J.B. and Poznyak, A.S. (2011). Convergence method, properties and computational complexity for Lyapunov games, International Journal of Applied Mathematics and Computer Science 21(2): 349-361, DOI: 10.2478/v10006-011-0026-x.

Clempner, J.B. and Poznyak, A.S. (2014). Simple computing of the customer lifetime value: A fixed local-optimal policy approach, Journal of Systems Science and Systems Engineering 23(4): 439-459.

De Fraja, G. and Delbono, F. (1990). Game theoretic models of mixed oligopoly, Journal of Economic Surveys 4(1): 1-17.

Harris, R. and Wiens, E. (1980). Government enterprise: An instrument for the internal regulation of industry, Canadian Journal of Economics 13(1): 125-132.

Karpowicz, M.P. (2012). Nash equilibrium design and price-based coordination in hierarchical systems, International Journal of Applied Mathematics and Computer Science 22(4): 951-969, DOI: 10.2478/v10006-012-0071-0.

Merril, W. and Schneider, N. (1966). Government firms in oligopoly industries: A short-run analysis, Quarterly Journal of Economics 80(5): 400-412.

Moya, S. and Poznyak, A.S. (2009). Extraproximal method application for a Stackelberg-Nash equilibrium calculation in static hierarchical games, IEEE Transactions on Systems, Man, and Cybernetics, B: Cybernetics 39(6): 1493-1504.

Nett, L. (1993). Mixed oligopoly with homogeneous goods, Annals of Public and Cooperative Economics 64(3): 367-393.

Nowak, P. and Romaniuk, M. (2013). A fuzzy approach to option pricing in a Levy process setting, International Journal of Applied Mathematics and Computer Science 23(3): 613-622, DOI: 10.2478/amcs-2013-0046.

Poznyak, A.S. (2009). Advance Mathematical Tools for Automatic Control Engineers, Vol. 1: Deterministic Techniques, Elsevier, Amsterdam.

Poznyak, A.S., Najim, K. and Gomez-Ramirez, E. (2000). Selflearning Control of Finite Markov Chains, Marcel Dekker, Inc., New York, NY.

Tanaka, K. and Yokoyama, K. (1991). On $\epsilon$-equilibrium point in a noncooperative n-person game, Journal of Mathematical Analysis and Applications 160(2): 413-423.

Trejo, K.K., Clempner, J.B. and Poznyak, A.S. (2015). A stackelberg security game with random strategies based on the extraproximal theoretic approach, Engineering Applications of Artificial Intelligence 37: 145-153.

Vickers, J. and Yarrow., G. (1998). Privatization-An Economic Analysis, MIT Press, Cambridge, MA.

von Stackelberg, H. (1934). Marktform und Gleichgewicht, Springer, Vienna. 


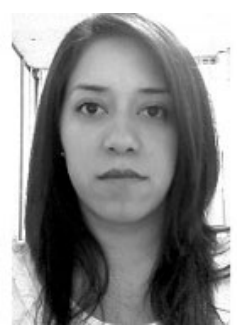

Kristal K. Trejo holds an M.Sc. from the Department of Automatic Control at the Center for Research and Advanced Studies (CINVESTAVIPN), Mexico. She received her B.Sc. in communications and electronic engineering from the Autonomous University of Zacatecas, Mexico. She follows a line of investigation focused on game theory and optimization research.

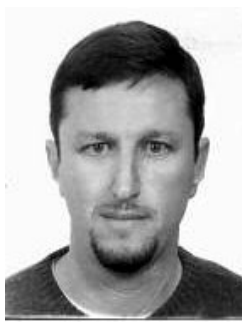

Julio B. Clempner has more than ten years of experience in the field of management consulting. He holds a Ph.D. in computer science from the Center for Computing Research at the National Polytechnic Institute. He specializes in applications of high technology related to project management, analysis and design of software, development of software (ad-hoc and products), information technology strategic planning, evaluation of software, business process reengineering and IT government, for infusing advanced computing technologies into diverse lines of businesses. Dr. Clempner's research interests are focused on justifying and introducing the Lyapunov equilibrium point in game theory. He is a member of the Mexican National System of Researchers (SNI), and of several North American and European professional organizations. Dr. Julio Clempner is on the editorial board of the International Journal of Applied Mathematics and Computer Science.

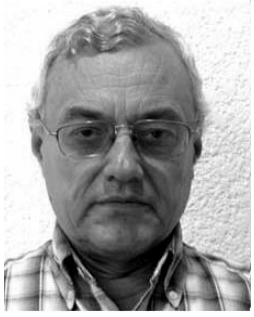

Alexander Poznyak graduated from Moscow Physical Technical Institute (MPhTI) in 1970 He earned his Ph.D. and Doctor's degrees from the Institute of Control Sciences of the Russian Academy of Sciences in 1978 and 1989, respectively. From 1973 up to 1993 he served this institute as a researcher and a leading researcher, and in 1993 he accepted the post of a full professor (3-E) at CINVESTAV of IPN in Mexico. Presently, he is the head of the Automatic Control Department. He was the supervisor of 35 Ph.D. theses (28 in Mexico). He has published more than 180 papers in various international journals and 10 books. He is a regular member of the Mexican Academy of Sciences and the System of National Investigators (SNI -3). He is a fellow of the IMA (Institute of Mathematics and Its Applications, Essex, UK) and an associate editor of Oxford-IMA Journal on Mathematical Control and Information, Kybernetika as well as the Iberamerican International Journal on Computations and Systems. He was also an associate editor of the Conference on Decision and Control (CDC), the American Control Conference $(A C C)$ and a member of the editorial board of the IEEE Control Systems Society (IEEE CSS).

Received: 21 March 2014

Revised: 15 November 2014 